\title{
Genome-wide analysis and expression profiling of glyoxalase gene families in soybean (Glycine max) indicate their development and abiotic stress specific response
}

\author{
Ajit Ghosh ${ }^{1^{*}}$ and Tahmina Islam²
}

\begin{abstract}
Background: Glyoxalase pathway consists of two enzymes, glyoxalase I (GLYI) and glyoxalase II (GLYII) which detoxifies a highly cytotoxic metabolite methylglyoxal (MG) to its non-toxic form. MG may form advanced glycation end products with various cellular macro-molecules such as proteins, DNA and RNA; that ultimately lead to their inactivation. Role of glyoxalase enzymes has been extensively investigated in various plant species which showed their crucial role in salinity, drought and heavy metal stress tolerance. Previously genome-wide analysis of glyoxalase genes has been conducted in model plants Arabidopsis and rice, but no such study was performed in any legume species.

Results: In the present study, a comprehensive genome database analysis of soybean was performed and identified a total of putative $41 \mathrm{GLYI}$ and 23 GLYII proteins encoded by 24 and 12 genes, respectively. Detailed analysis of these identified members was conducted including their nomenclature and classification, chromosomal distribution and duplication, exon-intron organization, and protein domain(s) and motifs identification. Expression profiling of these genes has been performed in different tissues and developmental stages as well as under salinity and drought stresses using publicly available RNAseq and microarray data. The study revealed that GmGLYI-7 and GmGLYII-8 have been expressed intensively in all the developmental stages and tissues; while GmGLYI-6, GmGLYI-9, GmGLYI-20, GmGLYII-5 and GmGLYII-10 were highly abiotic stress responsive members.

Conclusions: The present study identifies the largest family of glyoxalase proteins to date with $41 \mathrm{GmGLYI}$ and 23 GmGLYII members in soybean. Detailed analysis of GmGLYI and GmGLYII genes strongly indicates the genome-wide segmental and tandem duplication of the glyoxalase members. Moreover, this study provides a strong basis about the biological role and function of GmGLYI and GmGLYII members in soybean growth, development and stress physiology.
\end{abstract}

Keywords: Glyoxalase, Glycine max, Abiotic stress, Functional divergence, Gene duplication, Microarray, Metal dependency, RNA seq-Atlas, Semiquantitative RT-PCR

\footnotetext{
* Correspondence: ajitghoshbd@gmail.com

${ }^{1}$ Department of Biochemistry and Molecular Biology, Shahjalal University of

Science and Technology, Sylhet 3114, Bangladesh

Full list of author information is available at the end of the article
} 


\section{Background}

The glyoxalase system is a two-enzyme driven pathway that detoxifies the highly cytotoxic compound, methylglyoxal (MG) to D-lactate. The detoxification is accomplished by the sequential action of two thiol-dependent enzymes; glyoxalase I (GLYI) and glyoxalase II (GLYII). In presence of reduced glutathione (GSH), MG is converted into hemithioacetal (HTA) spontaneously, and GLYI catalyses the isomerization of this HTA into S-Dlactoyl-glutathione (SLG). GLYII hydrolyses SLG into D-lactate and recycles back one molecule of GSH to the system [1]. Both, the formation of MG and the glyoxalase enzymes have been ubiquitously found in all organisms from Escherichia coli to Homo sapiens [2].

Besides its proposed role in the detoxification of MG as metabolic enzyme, glyoxalase enzymes have been reported to be involved in various other functions. Glyoxalase system protects human from various vascular complications of diabetes, such as nephropathy, retinopathy, neuropathy and cardiovascular disease by resisting the increased accumulation of MG [3]. Moreover, glyoxalase pathway has also been shown to be involved in different important cellular functions of human, such as cell division and proliferation, microtubule assembly and protection against oxoaldehydes toxicity [4]. For this, the pathway has been regarded as "marker for cell growth and division". Similarly, stress tolerance potential of glyoxalase has been reported in plant by numerous studies [5]. Transgenic plants over-expressing GLYI and/or GLYII were found to provide significant tolerance against multiple abiotic stresses including salinity, drought and heavy metal toxicity $[5,6]$. Thus MG and glyoxalases are considered as potential biomarkers for plant stress tolerance [7].

Glyoxalase proteins have been extensively characterized from different genera such as Escherichia coli, Homo sapiens, Saccharomyces cerevisiae, Arabidopsis thaliana and Oryza sativa [2]. Compared to other organisms, very little is known about plant glyoxalases. The first plant glyoxalase activity was reported from Douglas fir needles by Smits and Johnson [5]. Thereafter, presence of glyoxalase activity has been reported from various other plant species, such as rice, Arabidopsis, tomato, wheat, sugarcane, Brassica etc. [7]. Most of the genes of plant exist as family due to the expansion and gene duplication during the course of plant evolution [8]. Availability of the whole genome sequences has opened up the field to identify and characterize plant glyoxalase family substantially. According to in silico genome wide analyses of rice and Arabidopsis, there are eleven potential GLYI and three GLYII genes in rice; and eleven GLYI and five GLYII genes in Arabidopsis [1]. Expression analysis of all these genes have been performed in different developmental tissues and stages, and in response to multiple abiotic stresses using publicly available MPSS and microarray database. It has been observed that AtGLYI-3, OsGLYI-11, AtGLYII-2, AtGLYII-5, OsGLYII-2 and OsGLYII-3 showed constitutive expression in all the tissues and stages, while AtGLYI-8, OsGLYI-3, and OsGLYI-10 expressed only in seed [1]. On the other hand, AtGLYI-7, OsGLYI-11, AtGLYII-2 and OsGLYII-3 were the most stress inducible members [1].

Among these identified glyoxalase members, GLYII genes have been extensively studied from both rice and Arabidopsis but the research on GLYI is still very limited. To date, all five AtGLYII and three OsGLYII genes have been well characterized. Both OsGLYII-2 and OsGLYII-3 possessed typical GLYII enzymatic activity and overexpression of these genes in tobacco provides enhanced tolerance against salinity stress [9, 10]. However, OsGLYII-1, along with AtGLYII-5 showed functional divergence by possessing sulphur dioxygenase (SDO) activity instead of GLYII [11]. One of the rice GLYI, OsGLYI-11.2 have been studied extensively and found to possess $\mathrm{Ni}^{2+}$-dependent GLYI activity with stress modulation potential [12].

Soybean (Glycine max [L.] Merr.) is a legume plant of Papilionoideae family [13], major source of vegetable protein and edible oil. It also has the capacity to fix atmospheric nitrogen through symbioses [14]. However, production of soybean is under threat due to the unfavourable environmental stimuli such as drought, salinity and osmotic stresses $[15,16]$. All these stresses severely affect the overall plant development in all the stages from germination to flowering and reduce the productivity and seed quality of soybean. The yield has been reported to be reduced by about $40 \%$ in response to drought [15]. Thus, there is an urgent need to identify novel stress responsive soybean genes using the available genome database [14]. The soybean genome contains 46,430 predicted protein-coding genes which are $70 \%$ more than Arabidopsis. There have been two genome duplication events undergone in soybean at approximately 59 and 13 million years ago, that resulted a highly duplicated genome (more than $75 \%$ of the genes are duplicated) [14]. A lot of gene families have been studied in soybean, such as ERF, HD-Zip, WRKY, BURP, MADS-box, MYB, NAC, CYP [13, 17-22].

Genome wide analyses of glyoxalase gene family have been done in Arabidopsis and rice [1], but no such analysis has been performed in soybean in spite of having a handful genome sequences deposited in the publicly available database. Here, we present a detailed genomewide identification of soybean GLYI and GLYII genes, their phylogenetic relationship, chromosomal distribution, structural and expressional analysis. Present results indicate that soybean genome contains 41 GLYI and 23 
GLYII proteins, the largest family of glyoxalase known to date in any organism. Expression analysis of these genes based on publicly available microarray data indicates the differentially regulation of glyoxalase members in response to various developmental cues as well as stress treatments. In particular GmGLYI-6, GmGLYI-9 and GmGLYII-5 are most up-regulated stress responsive members that might resist MG accumulation in stress by interacting with other members. This study will facilitate the further investigation of soybean glyoxalase genes for the biological and molecular functions.

\section{Results}

Identification of GLYI and GLYII gene families in soybean Proteins having lactoylglutathione lyase domain (PF00903) have been classified as GLYI proteins and metallo-betalactamase domain (PF00753) have been classified as GLYII proteins [1]. Previously, glyoxalase proteins have been identified in two model plant genome, Arabidopsis and rice [1]. To identify all the putative members of the glyoxalase proteins in soybean, a BLASTP search of the soybean genome database G. $\max$ Wm82.a2.v1 (http://phytozome.jgi.doe.gov/pz/portal.html\#!search?sh ow=BLAST\&method=Org_Gmax) was performed using the previously characterized protein sequence as a query. GLYI proteins have been primarily identified using a previously reported soybean GLYI protein (GenBank: NM_001249223.1). Subsequently, each of the newly identified GLYI protein sequences has been used as a query sequence individually in BLASTP search of soybean genome database. Subsequent searching process was repeated until there was no new member documented. This search resulted in the identification of total 43 unique proteins. All these identified proteins were analyzed using Pfam to check the presence of unique lactoylglutathione lyase domain (PF00903). This analysis discarded two members due to the lack of lactoylglutathione lyase domain, and finally landed to a total of 41 soybean GLYI proteins which is greater than the previously reported Arabidopsis (22) and rice (19) GLYI proteins. These 41 GLYI proteins have been coded by 24 unique genes located on 13 different chromosomes (Fig. 1). They were identified and named as GmGLYI-1 to GmGLYI-24 following the nomenclature proposed previously [1] (Table 1).

Similarly, soybean GLYII proteins have been primarily identified using a previously characterized Brassica juncea GLYII protein (GenBank: AAO26580.1) as query and secondarily by the newly identified members. A total of 26 unique protein sequences have been identified and checked for the presence of unique metallobeta-lactamase domain (PF00753) using Pfam. Three of them didn't have this unique domain and were discarded from the list. Thus, a total of 23 soybean GLYII proteins have been confirmed which is greater than the previously reported Arabidopsis (9) and rice (4) GLYII family members. These 23 GLYII proteins have been coded by 12 unique genes located on ten different chromosomes (Fig. 1). They were named as GmGLYII-1 to GmGLYII-12 like GmGLYI genes (Table 2). In both GmGLYI and GmGLYII families, the number of proteins

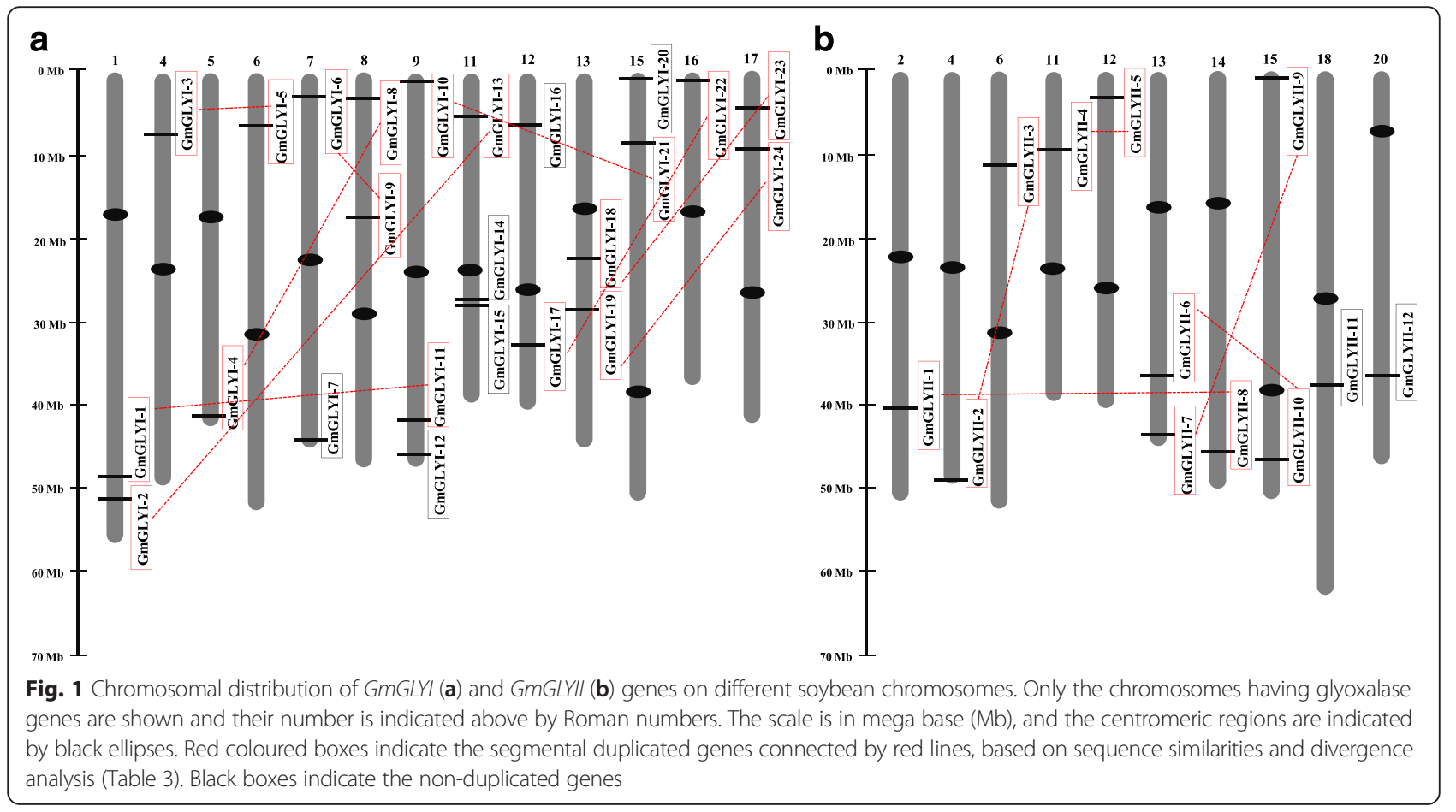


Table 1 List of identified GLYI genes in Soybean (Glycine max) along with their detailed information and localization

\begin{tabular}{|c|c|c|c|c|c|c|c|c|c|c|}
\hline Name & Gene & Protein & Chro. no & CDS coordinate ( $5^{\prime}$ to $\left.3^{\prime}\right)$ & CDS (bp) & Exons & PP length (aa) & $\mathrm{MW}(\mathrm{kDa})$ & $\mathrm{pl}$ & Localization \\
\hline \multirow[t]{3}{*}{ GmGLYI-1 } & \multirow[t]{3}{*}{ Glyma.01 g146300 } & Glyma.01 g146300.1 & \multirow[t]{3}{*}{1} & \multirow[t]{3}{*}{$48150827-48154366$} & 1044 & 9 & 347 & 39.3 & 7.01 & $\mathrm{Ch}^{\mathrm{a}, \mathrm{c}} ; \mathrm{Mt}^{\mathrm{b}} ; \mathrm{Cy}^{\mathrm{b}}$ \\
\hline & & Glyma.01 g146300.2 & & & 1038 & 9 & 345 & 39.1 & 7.01 & $\mathrm{Ch}^{\mathrm{a}, \mathrm{c}} ; \mathrm{Mt}^{\mathrm{b}} ; \mathrm{Cy}^{\mathrm{b}}$ \\
\hline & & Glyma.01 g146300.3 & & & 930 & 8 & 309 & 35.1 & 8.44 & $\mathrm{Ch}^{\mathrm{a}, \mathrm{b}, \mathrm{c}} ; \mathrm{Mt}^{\mathrm{b}} ; \mathrm{Cy}^{\mathrm{b}}$ \\
\hline GmGLYI-2 & Glyma.01 g168400 & Glyma.01 g168400.1 & 1 & $50605679-50608020$ & 579 & 3 & 192 & 22.0 & 5.04 & $C y^{a} ; E^{b}$ \\
\hline GmGLYI-3 & Glyma.04 g083100 & Glyma.04 g083100.1 & 4 & 7006088-7009361 & 1041 & 9 & 346 & 38.5 & 5.83 & $C h^{a, b, c}$ \\
\hline \multirow[t]{2}{*}{ GmGLYI-4 } & \multirow[t]{2}{*}{ Glyma.05 g228500 } & Glyma.05 g228500.1 & \multirow[t]{2}{*}{5} & \multirow[t]{2}{*}{$40646576-40651812$} & 1101 & 9 & 366 & 40.6 & 8.17 & $C h^{a, b, c}$ \\
\hline & & Glyma.05 g228500.2 & & & 1089 & 9 & 362 & 40.2 & 6.28 & $C h^{a, b, c}$ \\
\hline GmGLYI-5 & Glyma.06 g084500 & Glyma.06 g084500.1 & 6 & 6498718-6501298 & 792 & 8 & 263 & 29.7 & 5.45 & $C y^{b}$ \\
\hline \multirow[t]{3}{*}{ GmGLYI-6 } & \multirow[t]{3}{*}{ Glyma.07 g031700 } & Glyma.07 g031700.1 & \multirow[t]{3}{*}{7} & \multirow[t]{3}{*}{$2508024-2510223$} & 519 & 2 & 172 & 19.6 & 5.10 & $\mathrm{Nu}^{\mathrm{a}} ; \mathrm{Cy}^{\mathrm{b}}$ \\
\hline & & Glyma.07 g031700.2 & & & 369 & 3 & 122 & 13.9 & 4.89 & $C y^{a, b}$ \\
\hline & & Glyma.07 g031700.3 & & & 381 & 2 & 126 & 14.5 & 5.03 & $C y^{a, b}$ \\
\hline \multirow[t]{6}{*}{ GmGLYI-7 } & \multirow[t]{6}{*}{ Glyma.07 g261400 } & Glyma.07 g261400.1 & \multirow[t]{6}{*}{7} & \multirow[t]{6}{*}{$43645750-43649851$} & 843 & 7 & 280 & 31.6 & 5.62 & $C y^{a, b}$ \\
\hline & & Glyma.07 g261400.2 & & & 732 & 8 & 243 & 27.2 & 5.34 & $C y^{a, b}$ \\
\hline & & Glyma.07 g261400.3 & & & 795 & 7 & 264 & 29.8 & 5.24 & $C y^{a, b}$ \\
\hline & & Glyma.07 g261400.4 & & & 843 & 8 & 280 & 31.6 & 5.62 & $C y^{a, b}$ \\
\hline & & Glyma.07 g261400.5 & & & 843 & 8 & 280 & 31.6 & 5.62 & $C y^{a, b}$ \\
\hline & & Glyma.07 g261400.6 & & & 819 & 8 & 272 & 30.7 & 6.11 & $C y^{a, b}$ \\
\hline GmGLYI-8 & Glyma.08 g035400 & Glyma.08 g035400.1 & 8 & 2811954-2818455 & 1071 & 9 & 356 & 39.6 & 6.56 & $C h^{a, b, c}$ \\
\hline GmGLYI-9 & Glyma.08 g211100 & Glyma.08 g211100.1 & 8 & $17046316-17048640$ & 426 & 3 & 141 & 16.4 & 4.86 & $\mathrm{Ch}^{\mathrm{a}} ; \mathrm{Nu}^{\mathrm{a}} ; \mathrm{Cy}^{\mathrm{a}, \mathrm{b}}$ \\
\hline \multirow[t]{5}{*}{ GmGLYI-10 } & \multirow[t]{5}{*}{ Glyma.09 g004300 } & Glyma.09 g004300.1 & \multirow[t]{5}{*}{9} & \multirow[t]{5}{*}{$340275-344376$} & 975 & 8 & 324 & 36.9 & 6.62 & $\mathrm{Mt}^{\mathrm{a}} ; \mathrm{Cy}^{\mathrm{b}}$ \\
\hline & & Glyma.09 g004300.2 & & & 891 & 9 & 296 & 33.5 & 6.13 & $C y^{a, b}$ \\
\hline & & Glyma.09 g004300.3 & & & 864 & 9 & 287 & 32.4 & 5.74 & $C y^{a, b}$ \\
\hline & & Glyma.09 g004300.4 & & & 840 & 8 & 279 & 31.5 & 6.97 & $C y^{a, b}$ \\
\hline & & Glyma.09 g004300.5 & & & 951 & 9 & 316 & 35.9 & 8.45 & $\mathrm{Mt}^{\mathrm{a}, \mathrm{b}} ; \mathrm{Cy}^{\mathrm{b}}$ \\
\hline \multirow[t]{2}{*}{ GmGLYI-11 } & \multirow[t]{2}{*}{ Glyma.09 g193800 } & Glyma.09 g193800.1 & \multirow[t]{2}{*}{9} & \multirow[t]{2}{*}{$41830881-41834537$} & 1041 & 9 & 346 & 39.0 & 6.68 & $\mathrm{Ch}^{\mathrm{a}, \mathrm{b}, \mathrm{c}} ; \mathrm{Mt}^{\mathrm{b}}$ \\
\hline & & Glyma.09 g193800.2 & & & 819 & 7 & 272 & 30.6 & 8.76 & $\mathrm{Ch}^{\mathrm{a}, \mathrm{c}} ; \mathrm{Mt}^{\mathrm{b}}$ \\
\hline GmGLYI-12 & Glyma.09 g226500 & Glyma.09 g226500.1 & 9 & $45136253-45137166$ & 333 & 2 & 110 & 12.8 & 5.23 & $C y^{b}$ \\
\hline GmGLYI-13 & Glyma.11 g075000 & Glyma.11 g075000.1 & 11 & 5598647-5600229 & 579 & 3 & 192 & 22.1 & 4.97 & $C y^{a, b}$ \\
\hline GmGLYI-14 & Glyma.11 g194200 & Glyma.11 g194200.1 & 11 & $26776807-26779603$ & 747 & 8 & 248 & 28.5 & 5.48 & $\mathrm{Po}^{\mathrm{a}} ; \mathrm{Cy}^{\mathrm{b}} ; \mathrm{Ec}^{\mathrm{b}}$ \\
\hline GmGLYI-15 & Glyma.11 g194300 & Glyma.11 g194300.1 & 11 & $26780838-26784795$ & 702 & 8 & 233 & 26.5 & 9.16 & $\mathrm{Ch}^{\mathrm{a}, \mathrm{c}} ; \mathrm{Mt}^{\mathrm{b}}$ \\
\hline \multirow[t]{3}{*}{ GmGLYI-16 } & \multirow[t]{3}{*}{ Glyma.12 g079700 } & Glyma.12 g079700.1 & \multirow[t]{3}{*}{12} & $6241940-6246776$ & 708 & 7 & 235 & 26.8 & 9.28 & $M t^{a, b}$ \\
\hline & & Glyma.12 g079700.2 & & & 558 & 6 & 185 & 21.0 & 5.41 & $C y^{a, b}$ \\
\hline & & Glyma.12 g079700.3 & & & 525 & 8 & 174 & 20.0 & 9.69 & $M t^{a, b}$ \\
\hline
\end{tabular}


Table 1 List of identified GLYI genes in Soybean (Glycine max) along with their detailed information and localization (Continued)

\begin{tabular}{|c|c|c|c|c|c|c|c|c|c|c|}
\hline GmGLYI-17 & Glyma.12 g167400 & Glyma.12 g167400.1 & 12 & $32196920-32200101$ & 555 & 2 & 184 & 21.0 & 5.14 & $C y^{a, b} ; E c^{b}$ \\
\hline GmGLYI-18 & Glyma.13 g106600 & Glyma.13 g106600.1 & 13 & 22081599-22084230 & 630 & 5 & 209 & 23.4 & 6.49 & $\mathrm{Ch}^{\mathrm{a}, \mathrm{c}} ; \mathrm{Ec}^{\mathrm{b}}, \mathrm{Mt}^{\mathrm{b}}$ \\
\hline GmGLYI-19 & Glyma.13 g168200 & Glyma.13 g168200.1 & 13 & 28259866-28261852 & 504 & 3 & 167 & 19.0 & 5.46 & $\mathrm{EC}^{\mathrm{a}} ; \mathrm{Cy}^{\mathrm{b}} ; \mathrm{Nu}^{\mathrm{b}}$ \\
\hline GmGLYI-20 & Glyma.15 g009500 & Glyma.15 g009500.1 & 15 & 737953-739806 & 522 & 3 & 173 & 19.4 & 5.58 & $C h^{a, b} ; C y^{b}$ \\
\hline GmGLYI-21 & Glyma.15 g108400 & Glyma.15 g108400.1 & 15 & 8534420-8539477 & 864 & 8 & 287 & 32.4 & 5.74 & $C y^{a, b}$ \\
\hline GmGLYI-22 & Glyma.16 g003500 & Glyma.16 g003500.1 & 16 & 194687-196274 & 549 & 2 & 182 & 20.6 & 5.22 & $C y^{a, b} ; C h^{b}$ \\
\hline GmGLYI-23 & Glyma.17 g052700 & Glyma.17 g052700.1 & 17 & $4011185-4013445$ & 621 & 5 & 206 & 22.9 & 7.08 & $\mathrm{Ch}^{\mathrm{a}, \mathrm{c}} ; \mathrm{Mt}^{\mathrm{b}}$ \\
\hline GmGLYI-24 & Glyma.17 g115900 & Glyma.17 g115900.1 & 17 & 9163290-9164088 & 438 & 4 & 145 & 16.5 & 6.88 & $\mathrm{Ch}^{\mathrm{a}}, \mathrm{EC}^{\mathrm{a}, \mathrm{b}} ; \mathrm{Nu}^{\mathrm{b}}$ \\
\hline
\end{tabular}

Abbreviations: CDS coding DNA sequence, Chro chromosome, $P$ P polypeptide length, $M W$ molecular weight, $P^{\prime}$ isoelectric point, bp base pair, aa amino acid, $k D a$ kilodalton, Ch chloroplast, $C y$ cytosol, Ec extracellular, Mt mitochondria, Nu nucleus, Po peroxisome

aLocalization prediction by CELLO v.2.5 (http://cello.life.nctu.edu.tw/)

${ }^{\mathrm{b} L o c a l i z a t i o n}$ prediction by pSORT (http://www.genscript.com/wolf-psort.html)

cChloroplast localization signal confirmed by ChloroP (http://www.cbs.dtu.dk/services/ChloroP/) 
Table 2 List of identified GLYII genes in Soybean (Glycine max) along with their detailed information and localization

\begin{tabular}{|c|c|c|c|c|c|c|c|c|c|c|}
\hline Name & Gene & Protein & Chromosome no & CDS coordinate ( $5^{\prime}$ to $\left.3^{\prime}\right)$ & CDS (bp) & Exons & PP length (aa) & MW (kDa) & $\mathrm{pl}$ & Localization \\
\hline GmGLYII-1 & Glyma.02 g220100 & Glyma.02 g220100.1 & 2 & $40797403-40800820$ & 609 & 5 & 202 & 22.8 & 5.62 & $\mathrm{Cy}^{\mathrm{b}}$ \\
\hline \multirow[t]{4}{*}{ GmGLYII-2 } & \multirow[t]{4}{*}{ Glyma.04 g224100 } & Glyma.04 g224100.1 & \multirow[t]{4}{*}{4} & \multirow[t]{4}{*}{ 49456049-49460172 } & 600 & 6 & 199 & 22.0 & 7.12 & $C y^{a, b}$ \\
\hline & & Glyma.04 g224100.2 & & & 546 & 6 & 181 & 20.0 & 6.54 & $\mathrm{Cy}^{\mathrm{a}} ; \mathrm{Ec}^{\mathrm{b}}$ \\
\hline & & Glyma.04 g224100.3 & & & 486 & 5 & 161 & 17.9 & 6.49 & $C y^{a, b}$ \\
\hline & & Glyma.04 g224100.4 & & & 432 & 4 & 143 & 15.9 & 7.59 & $\mathrm{Cy}^{\mathrm{a}}, \mathrm{Ec}^{\mathrm{b}}$ \\
\hline GmGLYII-3 & Glyma.06 g140800 & Glyma.06 g140800.1 & 6 & 11478165-11482810 & 777 & 7 & 258 & 28.7 & 6.86 & $C y^{b}$ \\
\hline \multirow[t]{2}{*}{ GmGLYII-4 } & \multirow[t]{2}{*}{ Glyma.11 g126200 } & Glyma.11 g126200.1 & \multirow[t]{2}{*}{11} & \multirow[t]{2}{*}{ 9591802-9595913 } & 948 & 7 & 315 & 34.7 & 6.06 & $\mathrm{Nu}^{\mathrm{a}} ; \mathrm{Mt}^{\mathrm{b}}$ \\
\hline & & Glyma.11 g126200.2 & & & 861 & 7 & 286 & 31.5 & 5.92 & $C h^{a, b} ; M t^{a, b}$ \\
\hline \multirow[t]{3}{*}{ GmGLYII-5 } & \multirow[t]{3}{*}{ Glyma.12 g050800 } & Glyma.12 g050800.1 & \multirow[t]{3}{*}{12} & \multirow[t]{3}{*}{ 3651594-3655661 } & 951 & 8 & 316 & 35.0 & 5.93 & $\mathrm{Ch}^{\mathrm{a}} ; \mathrm{Mt}^{\mathrm{b}}$ \\
\hline & & Glyma.12 g050800.2 & & & 948 & 8 & 315 & 34.9 & 5.94 & $\mathrm{Nu}^{\mathrm{a}} ; \mathrm{Mt}^{\mathrm{b}}$ \\
\hline & & Glyma.12 g050800.3 & & & 924 & 8 & 307 & 34.0 & 6.22 & $\mathrm{Nu}^{\mathrm{a}} ; \mathrm{Mt}^{\mathrm{b}}$ \\
\hline GmGLYII-6 & Glyma.13 g261400 & Glyma.13 g261400.1 & 13 & $36531853-36536326$ & 1134 & 9 & 377 & 41.4 & 8.87 & $\mathrm{Ch}^{\mathrm{a}} ; \mathrm{PM}^{\mathrm{b}}, \mathrm{EC}^{\mathrm{b}}$ \\
\hline \multirow[t]{2}{*}{ GmGLYII-7 } & \multirow[t]{2}{*}{ Glyma.13 g345400 } & Glyma.13 g345400.1 & \multirow[t]{2}{*}{13} & \multirow[t]{2}{*}{$43601121-43604841$} & 990 & 7 & 329 & 36.3 & 8.82 & $\mathrm{Ch}^{\mathrm{a}, \mathrm{c}} ; \mathrm{Mt}^{\mathrm{b}}$ \\
\hline & & Glyma.13 g345400.2 & & & 876 & 8 & 291 & 32.0 & 7.71 & $M t^{a, b}$ \\
\hline \multirow[t]{2}{*}{ GmGLYII-8 } & \multirow[t]{2}{*}{ Glyma.14 g187700 } & Glyma.14 g187700.1 & \multirow[t]{2}{*}{14} & \multirow[t]{2}{*}{$45250598-45254278$} & 777 & 7 & 258 & 28.7 & 5.65 & $C y^{a, b}$ \\
\hline & & Glyma.14 g187700.2 & & & 546 & 5 & 181 & 20.0 & 5.84 & $C y^{a, b}$ \\
\hline \multirow[t]{2}{*}{ GmGLYII-9 } & \multirow[t]{2}{*}{ Glyma.15 g028900 } & Glyma.15 g028900.1 & \multirow[t]{2}{*}{15} & \multirow[t]{2}{*}{ 2325622-2329211 } & 981 & 7 & 326 & 35.8 & 9.0 & $\mathrm{Ch}^{\mathrm{a}, \mathrm{c}} ; \mathrm{Mt}^{\mathrm{b}}$ \\
\hline & & Glyma.15 g028900.2 & & & 948 & 8 & 315 & 34.7 & 8.88 & $\mathrm{Ch}^{\mathrm{a}, \mathrm{c}} ; \mathrm{Mt}^{\mathrm{b}}$ \\
\hline GmGLYII-10 & Glyma.15 g245500 & Glyma.15 g245500.1 & 15 & $46785385-46788016$ & 570 & 5 & 189 & 20.8 & 9.03 & $M t^{b} ; \mathrm{Ec}^{\mathrm{b}}$ \\
\hline GmGLYII-11 & Glyma.18 g163500 & Glyma.18 g163500.1 & 18 & $37294122-37294499$ & 258 & 2 & 85 & 9.5 & 6.34 & $\mathrm{Cy}^{\mathrm{b}} ; \mathrm{Ec}^{\mathrm{b}}$ \\
\hline \multirow[t]{3}{*}{ GmGLYII-12 } & \multirow[t]{3}{*}{ Glyma.20 g118000 } & Glyma.20 g118000.1 & \multirow[t]{3}{*}{20} & \multirow[t]{3}{*}{$36083279-36091841$} & 1584 & 12 & 527 & 58.8 & 6.56 & $\mathrm{Ch}^{\mathrm{a}} ; \mathrm{Cy}^{\mathrm{b}}$ \\
\hline & & Glyma.20 g118000.2 & & & 1467 & 12 & 488 & 54.5 & 5.92 & $\mathrm{Ch}^{\mathrm{a}} ; \mathrm{Cy}^{\mathrm{b}}$ \\
\hline & & Glyma.20 g118000.3 & & & 1467 & 12 & 488 & 54.5 & 5.92 & $\mathrm{Ch}^{\mathrm{a}} ; \mathrm{Cy}^{\mathrm{b}}$ \\
\hline
\end{tabular}

Abbreviations: CDS coding DNA sequence, $P P$ polypeptide length, $M W$ molecular weight, $P^{\prime}$ isoelectric point, $b p$ base pair, aa amino acid, $k D a$ kilodalton, $C h$ chloroplast, $C y$ cytosol, Ec extracellular, $M t$ mitochondria, $\mathrm{Nu}$ nucleus

aLocalization prediction by CELLO v.2.5 (http://cello.life.nctu.edu.tw/)

b Localization prediction by pSORT (http://www.genscript.com/wolf-psort.html)

chloroplast localization signal confirmed by ChloroP (http://www.cbs.dtu.dk/services/ChloroP/) 
was greater than the number of genes (Tables 1 and 2); indicating the existence of alternate splicing event in soybean glyoxalase genes. Most of the GmGLYI genes (17 out of 24) and GmGLYII genes (5 out of 12) showed only a single product. However, rest seven GmGLYI genes formed 24 alternative spliced products, whereas seven GmGLYII genes lead to the generation of 18 proteins (Tables 1 and 2).

\section{Detailed analysis of identified GmGLYI and GmGLYII members}

All the newly identified GmGLYI and GmGLYII members were analyzed in detail. The coding DNA sequence (CDS) length of the GmGLYI members vary from $333 \mathrm{bp}$ (GmGLYI-12.1) to 1101 bp (GmGLYI-4.1) with an average of 740 bp. Consequently, GmGLYI-4.1 encodes for the largest protein of the family with a polypeptide length of 366 aa and molecular weight of $40.6 \mathrm{kDa}$; and the smallest protein (GmGLYI-12.1) is 110 aa in length with $12.8 \mathrm{kDa}$ in weight (Table 1). Similar to the length and molecular weight variation, the proteins showed a wide range of deviation in their isoelectric point (pI) value from 4.86 (GmGLYI-9.1) to 9.69 (GmGLYI-16.3). Most of the GmGLYI members showed acidic pI value (less than or around 7), with only seven such as GmGLYI-1.3, GmGLYI-4.1, GmGLYI-10.5, GmGLYI11.2, GmGLYI-15.1, GmGLYI-16.1, and GmGLYI-16.3 have showed basic pI value (Table 1). This ensures the presence of both positively and negatively charged GmGLYI proteins at a certain physiological condition. Sub-cellular localization of all these predicted GmGLYI proteins (41) were analyzed based on two different tools CELLO [23] and Wolf pSORT [24], and the chloroplast localization was further confirmed by ChloroP [25]. Different members were found to be localized at different sub-cellular compartments, such as chloroplast, cytosol, mitochondria, nucleus, extracellular, peroxisome. Most of the GmGLYI proteins are found to be localized in cytosol, followed by chloroplast, mitochondria and nucleus (Table 1).

Similarly, the CDS length of GmGLYII transcripts varies from $432 \mathrm{bp}$ (GmGLYII-2.4) to 1584 bp (GmGLYII12.1) with an average of $850 \mathrm{bp}$ (Table 2). The largest GmGLYII-12.1 protein is 527 aa in length with a molecular weight of $58.8 \mathrm{kDa}$; and the smallest protein (GmGLYII-2.4) is 143 aa in length and $15.9 \mathrm{kDa}$ in weight (Table 2). GmGLYII proteins also show variation in their $\mathrm{pI}$ values ranging from 5.62 (GmGLYII-1.1) to 9.03 (GmGLYII-10.1). Most of the GmGLYII members (15 out of 23) showed acidic PI value similar to GmGLYI proteins, while only eight GmGLYII members such as GmGLYII-2.1, GmGLYII-2.4, GmGLYII-6.1, GmGLYII7.1, GmGLYII-7.2, GmGLYII-9.1, GmGLYII-9.2, and GmGLYII-10.1 have basic pI value (Table 2). Similar to
GmGLYI, most of the GmGLYII proteins are found to be localized in cytosol, followed by chloroplast (4), nucleus (3), and mitochondria (2).

\section{Chromosomal distribution and gene duplication}

To determine the exact position and distribution of the identified GmGLYI and GmGLYII genes on different chromosomes, a detailed chromosome map was constructed. Soybean glyoxalase genes are found to be unevenly distributed throughout the chromosomes. It has been found that 24 GmGLYI genes are located on 13 different chromosomes (Fig. 1a). The gene density per chromosome is highly uneven, where Chromosome 9 and 11 contain the maximum occurrence of GLYI genes (3 each). However, chromosomes 1, 7, 8, 12, 13, 15, 18 have two GLYI genes each, and only one GLYI gene each is present on chromosomes 4, 5, 6, and 16. No GLYI gene was found on chromosomes 2, 3, 10, 14, 18, 19 and 20; thereafter not shown in the Fig. 1a. Similarly, 12 GmGLYII genes were found to be located on ten different chromosomes (Fig. 1b) and the gene density per chromosome is highly uneven. Chromosomes 13 and 15 contain the maximum GLYII genes (2 each), whereas chromosomes $2,4,6,11,12,14,18$, and 20 have only one GLYII gene each. No GLYII gene was found on the rest of the chromosomes and as such not shown in the Fig. 1b. All the GmGLYI and GmGLYII genes were found to be located towards the chromosome ends (Fig. 1), suggesting the possibility of inter-chromosomal genetic rearrangements between different soybean chromosomes during genome duplication.

Due to two duplication events, soybean genome resulted in many paralogs within a gene family [14]. Out of the 24 GmGLYI proteins (only the first member in case of different alternate splice form), 20 are clustered in pairs (10 pairs) and eight GmGLYII proteins are clustered in pairs (4 pairs) out of a total of 12 GmGLYII proteins in the phylogenetic tree (Additional file 1: Figure S1). The percentage of similarities between all these GmGLYI (Additional file 2: Table S1) and GmGLYII (Additional file 2: Table S2) proteins were combined separately. It was observed that all the paired members of both GLYI and GLYII family (GmGLYI-1/-11, GmGLYI-4/-8, GmGLYI-10/-21, GmGLYI-3/-5, GmGLYI14/-15, GmGLYI-18/-23, GmGLYI-2/-13, GmGLYI-17/22, GmGLYI-19/-24 and GmGLYI-6/-9; GmGLYII-4/-5, GmGLYII-9/-11, GmGLYII-2/-3, GmGLYII-6/-10) have very high level (more than $90 \%$ ) of sequence similarities. This high level of sequence similarities indicates the possibility of segmental duplication of the genes throughout evolution. Moreover, among the 24 GmGLYI genes one gene pair (GmGLYI-14 and GmGLYI-15) was present continuously (without any gene in between) within a distance of less than $5 \mathrm{~kb}$ (1200 bp exactly) on 
chromosome 11. This indicates that these two genes might be duplicated by tandem duplication (Fig. 1). To identify the time course of gene duplication, all the identified duplicated gene pairs were analyzed using plant genome duplication database (http://chibba.agtec.uga.edu/ duplication/index/downloads) [26] (Table 3). According to the ratio of nonsynonymous to synonymous substitutions $(\mathrm{Ka} / \mathrm{Ks})$, the evolutionary history of selection acting on different genes could be measured [17, 27]. This ratio could be used to interpret the direction and magnitude of natural selection enforcing on the various protein coding genes. A pair of sequences having $\mathrm{Ka} / \mathrm{Ks}<1$ implies purifying selection; $\mathrm{Ka} / \mathrm{Ks}=1$ indicates both sequences are drifting neutrally; and lastly $\mathrm{Ka} / \mathrm{Ks}>1$ implies positive or Darwinian selection [17, 28]. The $\mathrm{Ka} / \mathrm{Ks}$ of 15 glyoxalase duplicated gene pairs (Table 3) was found to be less than 0.55 ; that indicates the influence of purifying selection in the evolution of these gene pairs. Considering the divergence rate of 6.161029 synonymous mutations per synonymous site per year for soybean [29], the duplication time for each gene pairs was calculated. It is observed that all the segmental duplicated pairs showed a time frame between 3.7 and $18.8 \mathrm{Mya}$, except the tandem duplicated pair that occurred 33.9 Mya ago (Table 3).

\section{Phylogenetic analysis of glyoxalase genes from various plant species}

In the present study, a phylogenetic tree of all the identified GmGLYI or GmGLYII proteins along with other reported GLYI or GLYII proteins from other plant species were constructed using Mega 5.2 tool (Fig. 2). A neighbour joining phylogenetic tree was generated using a total of 83 full-length GLYI protein sequences of soybean, rice and Arabidopsis GLYI family, and proteins from other plant species. The tree was sub-divided into four subfamilies (I to IV) as evident in Fig. 2a. All these subfamilies consist of representative member from both dicot Arabidopsis and monocot rice, indicating that the evolution of plant GLYI genes occurred before the split of dicotmonocot. Clade-IV has the largest GLYI members from different plant species, while clade-II has the lowest number of members only from Arabidopsis and rice genome (Fig. 2a). Clade-I comprises of GLYI members only from the complete genome database of three plants, Arabidopsis, rice and soybean. Among them, OsGLYI-10 is functionally a diverge member of the rice GLYI family and might possess some other activities than GLYI (unpublished data). In clade-III, there are multiple members from Arabidopsis, rice and soybean; and one member each from Genlisea aurea and Sorghum bicolor. Among them, three rice members OsGLYI-2, OsGLYI-7 and OsGLYI-11; and two members of Arabidopsis AtGLYI-3 and AtGLYI-6 have been already predicted to be $\mathrm{Ni}^{2+}$-dependent GLYI enzyme [2]. Thus rest of the members of this clade would be expected to have $\mathrm{Ni}^{2+}$-dependent catalytic activity. Similarly, clade-IV has members from rice (OsGLYI-8) and Arabidopsis (AtGLYI-2) which are $\mathrm{Zn}^{2+}$-dependent GLYI enzymes [2]. Thus rest of the GLYI members from other species would require $\mathrm{Zn}^{2+}$ for their optimum GLYI activity. This indicates that $\mathrm{Zn}^{2+}$-dependent GLYI enzymes are more diverse as they are present in many plant species (Fig. 2a).

To clarify the phylogenetic relationship among GLYII proteins, we further constructed another tree for all full length sequences of GmGLYII, OsGLYII, AtGLYII family and GLYII sequences from other plant species (Fig. 2b).

Table 3 Divergence time between glyoxalase gene pairs in Soybean

\begin{tabular}{|c|c|c|c|c|c|c|c|}
\hline SI. no & Locus 1 & Locus 2 & ka & ks & $\mathrm{ka} / \mathrm{ks}$ & Duplication time (Mya) & Duplication type \\
\hline 1 & GmGLYI-1 & GmGLYI-11 & 0.0354 & 0.1246 & 0.2841 & 10.2131 & Segmental \\
\hline 2 & GmGLYI-2 & GmGLYI-13 & 0.0066 & 0.0983 & 0.0671 & 8.0574 & Segmental \\
\hline 3 & GmGLYI-3 & GmGLYI-5 & 0.0317 & 0.0823 & 0.3852 & 6.7459 & Segmental \\
\hline 4 & GmGLYI-4 & GmGLYI-8 & 0.0451 & 0.1094 & 0.4122 & 8.9672 & Segmental \\
\hline 5 & GmGLYI-6 & GmGLYI-9 & 0.0418 & 0.1581 & 0.2644 & 12.9590 & Segmental \\
\hline 6 & GmGLYI-10 & GmGLYI-21 & 0.0076 & 0.0455 & 0.1670 & 3.7295 & Segmental \\
\hline 7 & GmGLYI-14 & GmGLYI-15 & .2260 & 0.4137 & 0.5463 & 33.9098 & Tandem \\
\hline 8 & GmGLYI-17 & GmGLYI-22 & 0.0263 & 0.1434 & 0.1834 & 11.7541 & Segmental \\
\hline 9 & GmGLYI-18 & GmGLYI-23 & 0.0839 & 0.1666 & 0.5036 & 13.6557 & Segmental \\
\hline 10 & GmGLYI-19 & GmGLYI-24 & 0.0450 & 0.1442 & 0.3121 & 11.8197 & Segmental \\
\hline 11 & GmGLYII-1 & GmGLYII-8 & 0.0804 & 0.1449 & 0.5549 & 11.8770 & Segmental \\
\hline 12 & GmGLYII-2 & GmGLYII-3 & 0.1142 & 0.1502 & 0.7603 & 12.3115 & Segmental \\
\hline 13 & GmGLYII-4 & GmGLYII-5 & 0.0278 & 0.1353 & 0.2055 & 11.0902 & Segmental \\
\hline 14 & GmGLYII-6 & GmGLYII-10 & 0.0916 & 0.229 & 0.4000 & 18.7705 & Segmental \\
\hline 15 & GmGLYII-7 & GmGLYII-9 & 0.0246 & 0.0767 & 0.3207 & 6.2869 & Segmental \\
\hline
\end{tabular}



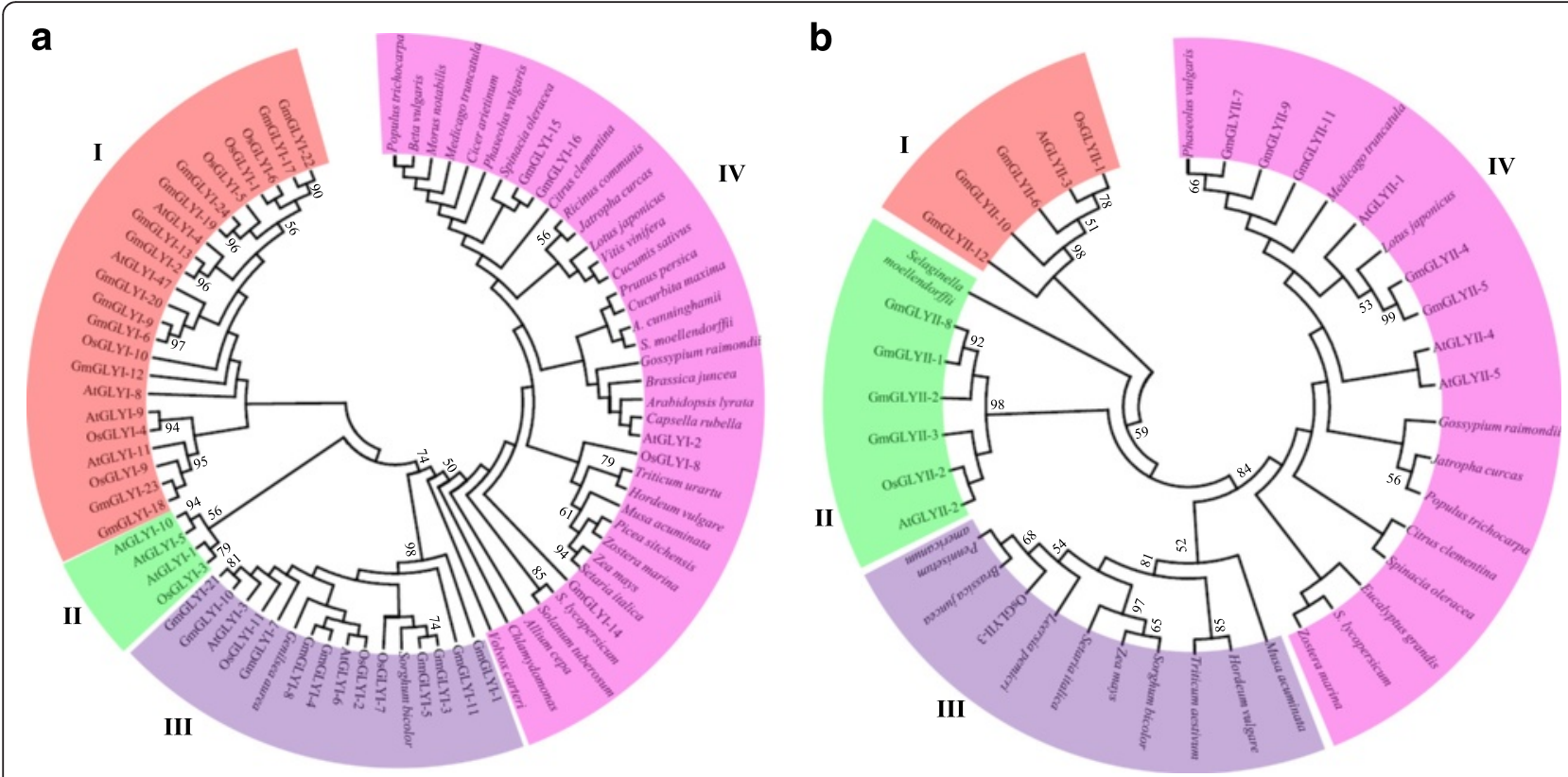

Fig. 2 Phylogenetic analyses of GLYI (a) and GLYII (b) proteins from various plant species. Glyoxalase protein sequences from various plant species were downloaded from various databases and provided as Additional files 4 and 5. An unrooted tree was generated using Neighbor-Joining method with 1000 bootstrap by MEGA5.2 software using the full-length amino acid sequences of eighty-three GLYl (a) or forty-one GLYII (b) proteins (only the first splice variants were taken in case of multiple splice forms). The numbers next to the branch shows the result of 1000 bootstrap replicates expressed in percentage, and scores higher than $50 \%$ are indicated on the nodes. Both trees were sub-divided into four classes (marked by I to IV) and indicated by different colours

This tree was subdivided into four classes (I to IV) too like the previous one. Class-I consists of three proteins from soybean, and one each from rice (OsGLYII-1) and Arabidopsis (AtGLYII-3). Among them, OsGLYII-1 has been reported to have sulphur dioxygenase (SDO) activity rather than GLYII [11]. So this sub class of proteins would be functionally diverse from GLYII. Similarly, class-II contains one protein each from rice (OsGLYII-2), Arabidopsis (AtGLYII-2), and Selaginella moellendorffii, and four proteins from soybean. AtGLYII-2 has been reported to be the mitochondrial localized AtGLYII family member [30]. Division of class-III and -IV is more interesting and evolutionarily more significant. Class-III has GLYII proteins from all monocot plants (rice, Zea mays, Pennisetum, Brassica, Triticum, Hordeum); while class-IV has exclusively dicot members including Arabidopsis, soybean, Medicago, lotus etc. (Fig. 2b). Apart from GLYI, GLYII proteins were found to be diversified after the split of monocot and dicot.

\section{Gene structures of GmGLYI and GmGLYII genes}

Detailed analysis of the exon-intron structure of GmGLYI (Fig. 3a) and GmGLYII (Fig. 3b) genes showed great variation among themselves. All GmGLYI and GmGLYII genes contained at least one intron in their open reading frame (ORF), which means there is no intron less glyoxalase gene in soybean. The number of introns varied from 1 to 9 in the ORFs of different GmGLYI genes (Fig. 3a and Additional file 3: Table S3). The GmGLYI-6.3, GmGLYI-12, GmGLYI-17, and GmGLYI-22 contained a single intron in their ORF while the largest numbers of introns (9) were found in the GmGLYI-4.2 transcript. In many cases, the borders of protein-coding sequence, $5^{\prime}$ and $3^{\prime}$ untranslated regions (UTR) also contain large numberof introns [13, 31]. Out of 41 GmGLYI transcripts, there was no intron in the 3' UTR of any of these genes and only eight of them contained a single intron in their $5^{\prime}$ UTR region. Similarly, the number of introns varied from 1 to 12 in the ORFs of different GmGLYII genes (Fig. 3b and Additional file 3: Table S4). The maximum number of introns (12) was observed in GmGLYII-12.1, followed by 11 each in GmGLYII-12.2 and GmGLYII-12.3. GmGLYII-11.1 contained only a single intron in its ORF while the rest have varied number of introns. Similar to GmGLYI transcripts, there was no intron in the 3' UTR of GmGLYII transcripts. Only six out of 23 transcripts (GmGLYII-2.2, GmGLYII-2.4, GmGLYII-4.2, GmGLYII6.1, GmGLYII-7.1 and GmGLYII-12.1) have a single intron in their $5^{\prime} \mathrm{UTR}$ region.

Longer introns are selectively advantageous that could counterbalance the mutational bias and improve 


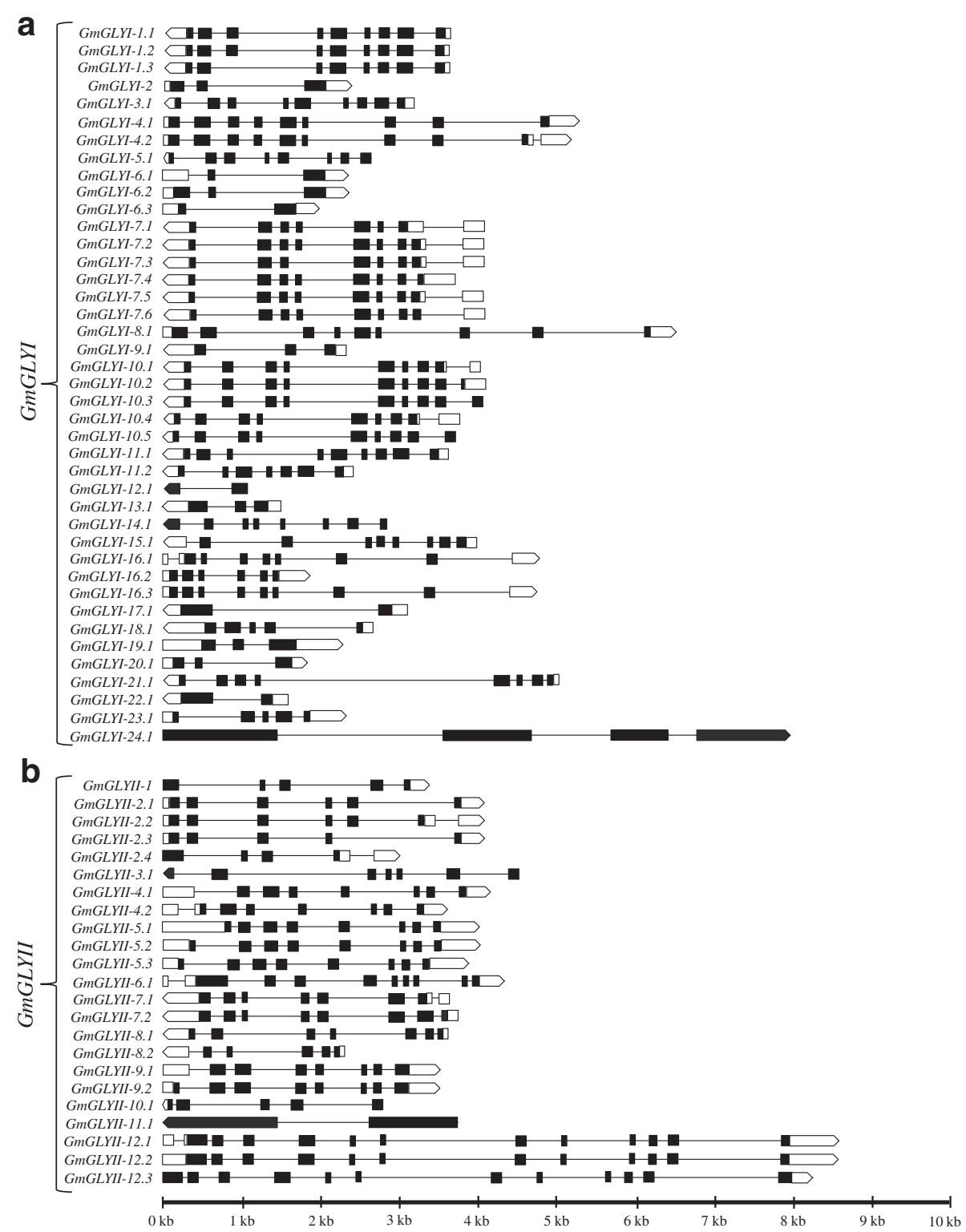

Fig. 3 Gene structures of GmGLYI (a) and GmGLYII (b) family members including the alternative spliced forms. All the exons are shown in filled black boxes and the introns are indicated by black lines. The 5'-UTR regions are shown using empty boxes and the 3'-UTR regions are shown in empty arrows which also indicate the direction of the gene. Left to right direction of transcript indicates " + " strand, while the right to left one indicates "-" strand, relative to the annotation of the genome sequence. The size of the introns, exons, and UTRs could be estimated from the scale at the bottom

the recombination frequency [32]. A strong evidence for the presence of ancestral introns was reported by analyzing introns of animal, plant and fungus [33]. Moreover, the number of exons and introns were found to be similar in the paralogous genes (Fig. 3) that clustered together in the phylogenetic analysis (Additional file 1: Figure S1). Such as, GmGLYI-1/-11, GmGLYI-4/8, GmGLYI-10/-21, and GmGLYI-6/-9 have the same number of introns and exons.
Analysis of GmGLYI proteins for their domain architecture, catalytic conservance and metal ion dependency

All the predicted GmGLYI (41) proteins were analyzed using Pfam to reveal the presence of conserved glyoxalase domain (PF00903) among them. Analyses of GmGLYI proteins revealed that 21 out of forty-one contains two GLYI domains, while the rest 20 have only single GLYI domain (Fig. 4). Presence of two GLYI domain in a single protein have been previously 


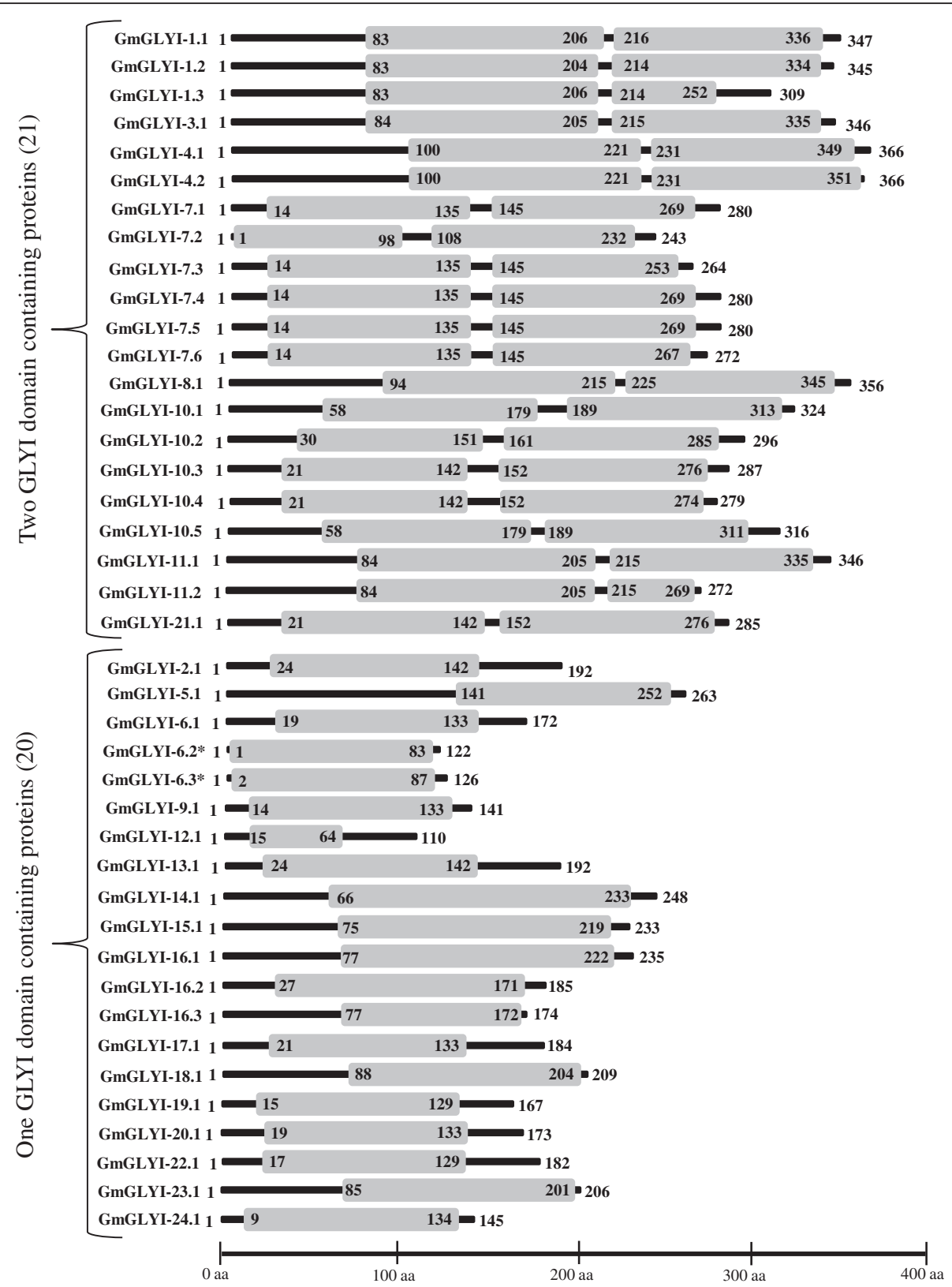

Fig. 4 Domain architectures of GmGLYI proteins. All forty-one soybean GLYI proteins were analyzed for the presence of functional domain(s) using pfam (http://pfam.xfam.org/). All the GmGLYI proteins possess glyoxalase domain (PF00903) that is represented by boxes. The position of the domain(s) is indicated by the amino acid number inside the box. Among the $41 \mathrm{GmGLYl} \mathrm{members,} 20$ of them have single glyoxalase domain, whereas rest 21 have two domains. The length of full proteins is indicated by exact amino acid numbers and relative position of the domains could be interpreted by the scale given below

reported from Saccharomyces cerevisiae [34], Oryza sativa [12] and Plasmodium falciparum [35]. Presence of two domain forms two putative active sites on a single monomeric protein. Both the active sites are found to be functional, but allosterically regulated in Plasmodium falciparum [35], whereas one of the active site is found to be a pseudo-active site in Oryza sativa [12]. However, GLYI proteins with single domain have also been reported from various species such as E. coli [36], H. sapiens [37] and function as homo-dimer.

Activity of GLYI enzyme is highly dependent on divalent metal ions [2]. On the basis of metal ion specificity GLYI proteins could be divided into two classes; $\mathrm{Zn}^{2+}$-dependent or $\mathrm{Zn}^{2+}$-independent (mainly $\mathrm{Ni}^{2+} / \mathrm{Co}^{2+}$-dependent). GLYI from Homo sapiens, Saccharomyces cerevisiae and Pseudomonas putida have been reported as $\mathrm{Zn}^{2+}$-dependent [38-40], whereas GLYI from E. coli and one of the rice 
GLYI (OsGLYI-11.2) showed $\mathrm{Ni}^{2+}$-dependent activity $[12,36]$. The metal dependency of the GLYI enzymes could be easily predicted from the length of GLYI domain, as $\mathrm{Ni}^{2+}$-dependent GLYI has a domain length of $\sim 120$ aa and $\mathrm{Zn}^{2+}$-dependent GLYIs are usually 142 aa in length [2]. Irrespective of the metal ion dependency, the active site of GLYI proteins has a conserved motif of $\mathrm{H} / \mathrm{QEH} /$ $\mathrm{QE}$. Among them, the glutamate residues act as a base by accepting protons from the substrate and any mutation of this conserved residue resulted in the complete loss of activity $[12,41]$. Thus, to comment on the presence of enzymatic activity and metal ion dependency, GLYI domain (only $\mathrm{N}$-terminal one in case of two domain containing members) of all the putative GmGLYI proteins were aligned (Fig. 5) along with known $\mathrm{Ni}^{2+}$-dependent OsGLYI-11.2 and $\mathrm{Zn}^{2+}$-dependent OsGLYI-8 [2] proteins. All the metal binding sites were presented inside black boxes and the regions specific for $\mathrm{Zn}^{2+}$-dependent GLYI were presented by black arrows (Fig. 5).

Based on the presence of all the four conserved metal binding site, the expected GLYI enzyme activity of the putative GmGLYI proteins was predicted (Table 4). Out of a total 41 putative GmGLYI proteins, 20 have all the four conserved residues and are expected to have functional GLYI enzyme activity (Fig. 5 and Table 4). Out of this 20 expected functional GLYI enzymes, 16 are predicted to be $\mathrm{Ni}^{2+}$-dependent as they have the domain length of around 120 aa and lack of the conserved regions specific for $\mathrm{Zn}^{2+}$-dependent members. The remaining four namely GmGLYI-14.1, GmGLYI-15.1, GmGLYI-16.1 and GmGLYI-16.2 are expected to be $\mathrm{Zn}^{2+}$-dependent as their domain length is more than 145 aa and possessed the conserved regions (Fig. 5 and Table 4).

\section{Analysis of GmGLYII proteins for their domain architecture and catalytic efficiency}

Genome wide analysis of soybean revealed the presence of 23 GLYII proteins coded by 12 genes (Table 2). All these GmGLYII proteins were analyzed using Pfam to reveal the presence of conserved metallo-beta-lactamase domain (PF00753) among them. Analysis of all GmGLYII proteins revealed that 12 out of 23 have only metallo-betalactamase domain, while the rest eleven contain additional Hydroxyacylglutathione hydrolase C-terminus (HAGH-C) domain (PF16123) along with metallo-beta-lactamase domain (Fig. 6). HAGH-C domain is usually found to be present at the C-terminus of GLYII enzymes that forms the substrate binding site along with the catalytic domain (PF00753) [42]. However, GLYII from various species such as $E$. coli, S. cerevisiae, S. typhimurium, $L$. infantum, A. thaliana, B. juncea, O. sativa and $H$. sapiens, contained the well conserved metal binding motif (THXHXDH) and active site motif (C/GHT) [9]. Both these motifs play an important role in the GLYII enzyme activity of a protein. Therefore, to comment on the presence of enzymatic activity of the putative

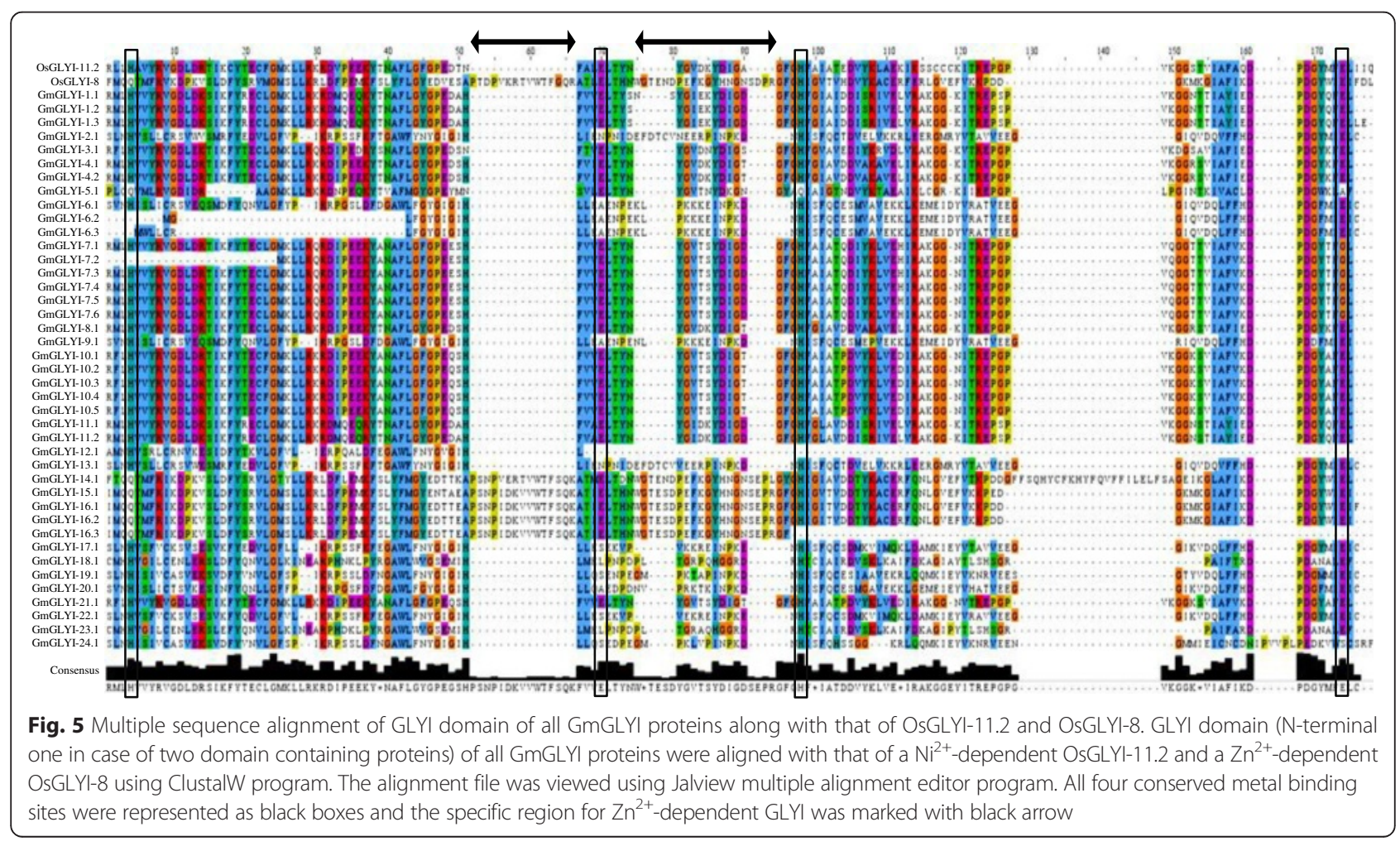


Table 4 Analysis of all putative GmGLYI enzymes for their enzymatic activity and metal ion dependency

\begin{tabular}{|c|c|c|c|c|c|c|c|c|}
\hline \multirow[t]{2}{*}{ Sl. no } & \multirow[t]{2}{*}{ Proteins } & \multicolumn{4}{|c|}{ Metal binding sites } & \multirow{2}{*}{$\begin{array}{l}\text { Expected GLYI } \\
\text { enzyme activity }\end{array}$} & \multirow{2}{*}{$\begin{array}{l}\text { Length of GLYI } \\
\text { domain (aa) }\end{array}$} & \multirow{2}{*}{$\begin{array}{l}\text { Metal ion } \\
\text { dependency }\end{array}$} \\
\hline & & $\mathrm{H} / \mathrm{Q}$ & $E$ & $\mathrm{H} / \mathrm{Q}$ & $E$ & & & \\
\hline 1 & GmGLYI-1.1 & $\sqrt{ }$ & $\sqrt{ }$ & $\sqrt{ }$ & $\sqrt{ }$ & Present & 124 & $\mathrm{Ni}$ \\
\hline 2 & GmGLYI-1.2 & $\sqrt{ }$ & $\sqrt{ }$ & $\sqrt{ }$ & $\sqrt{ }$ & Present & 122 & $\mathrm{Ni}$ \\
\hline 3 & GmGLYI-1.3 & $\sqrt{ }$ & $\sqrt{ }$ & $\sqrt{ }$ & $\sqrt{ }$ & Present & 124 & $\mathrm{Ni}$ \\
\hline 4 & GmGLYI-2.1 & $\sqrt{ }$ & $\sqrt{ }$ & $\sqrt{ }$ & $\sqrt{ }$ & Present & 125 & $\mathrm{Ni}$ \\
\hline 5 & GmGLYI-3.1 & $\sqrt{ }$ & $\sqrt{ }$ & $\sqrt{ }$ & $\sqrt{ }$ & Present & 122 & $\mathrm{Ni}$ \\
\hline 6 & GmGLYI-4.1 & $\sqrt{ }$ & $\sqrt{ }$ & $\sqrt{ }$ & $\sqrt{ }$ & Present & 122 & $\mathrm{Ni}$ \\
\hline 7 & GmGLYI-4.2 & $\sqrt{ }$ & $\sqrt{ }$ & $\sqrt{ }$ & $\sqrt{ }$ & Present & 122 & $\mathrm{Ni}$ \\
\hline 8 & GmGLYI-5.1 & $\sqrt{ }$ & $\sqrt{ }$ & $\sqrt{ }$ & - & Absent & 115 & - \\
\hline 9 & GmGLYI-6.1 & $\sqrt{ }$ & - & $\sqrt{ }$ & $\sqrt{ }$ & Absent & 121 & - \\
\hline 10 & GmGLYI-6.2 & - & - & $\sqrt{ }$ & $\sqrt{ }$ & Absent & 83 & - \\
\hline 11 & GmGLYI-6.3 & - & - & $\sqrt{ }$ & $\sqrt{ }$ & Absent & 87 & - \\
\hline 12 & GmGLYI-7.1 & $\sqrt{ }$ & $\sqrt{ }$ & $\sqrt{ }$ & - & Absent & 122 & - \\
\hline 13 & GmGLYI-7.2 & - & $\sqrt{ }$ & $\sqrt{ }$ & - & Absent & 98 & - \\
\hline 14 & GmGLYI-7.3 & $\sqrt{ }$ & $\sqrt{ }$ & $\sqrt{ }$ & - & Absent & 122 & - \\
\hline 15 & GmGLYI-7.4 & $\sqrt{ }$ & $\sqrt{ }$ & $\sqrt{ }$ & - & Absent & 122 & - \\
\hline 16 & GmGLYI-7.5 & $\sqrt{ }$ & $\sqrt{ }$ & $\sqrt{ }$ & - & Absent & 122 & - \\
\hline 17 & GmGLYI-7.6 & $\sqrt{ }$ & $\sqrt{ }$ & $\sqrt{ }$ & - & Absent & 122 & - \\
\hline 18 & GmGLYI-8.1 & $\sqrt{ }$ & $\sqrt{ }$ & $\sqrt{ }$ & $\sqrt{ }$ & Present & 122 & $\mathrm{Ni}$ \\
\hline 19 & GmGLYI-9.1 & $\sqrt{ }$ & - & $\sqrt{ }$ & $\sqrt{ }$ & Absent & 120 & - \\
\hline 20 & GmGLYI-10.1 & $\sqrt{ }$ & $\sqrt{ }$ & $\sqrt{ }$ & $\sqrt{ }$ & Present & 122 & $\mathrm{Ni}$ \\
\hline 21 & GmGLY|-10.2 & $\sqrt{ }$ & $\sqrt{ }$ & $\sqrt{ }$ & $\sqrt{ }$ & Present & 122 & $\mathrm{Ni}$ \\
\hline 22 & GmGLYI-10.3 & $\sqrt{ }$ & $\sqrt{ }$ & $\sqrt{ }$ & $\sqrt{ }$ & Present & 122 & $\mathrm{Ni}$ \\
\hline 23 & GmGLYI-10.4 & $\sqrt{ }$ & $\sqrt{ }$ & $\sqrt{ }$ & $\sqrt{ }$ & Present & 122 & $\mathrm{Ni}$ \\
\hline 24 & GmGLYI-10.5 & $\sqrt{ }$ & $\sqrt{ }$ & $\sqrt{ }$ & $\sqrt{ }$ & Present & 122 & $\mathrm{Ni}$ \\
\hline 25 & GmGLYI-11.1 & $\sqrt{ }$ & $\sqrt{ }$ & $\sqrt{ }$ & $\sqrt{ }$ & Present & 122 & $\mathrm{Ni}$ \\
\hline 26 & GmGLYI-11.2 & $\sqrt{ }$ & $\sqrt{ }$ & $\sqrt{ }$ & $\sqrt{ }$ & Present & 122 & $\mathrm{Ni}$ \\
\hline 27 & GmGLYI-12.1 & $\sqrt{ }$ & - & - & - & Absent & 50 & - \\
\hline 28 & GmGLYI-13.1 & $\sqrt{ }$ & - & $\sqrt{ }$ & $\sqrt{ }$ & Absent & 125 & - \\
\hline 29 & GmGLYI-14.1 & $\sqrt{ }$ & $\sqrt{ }$ & $\sqrt{ }$ & $\sqrt{ }$ & Present & 169 & $\mathrm{Zn}$ \\
\hline 30 & GmGLYI-15.1 & $\sqrt{ }$ & $\sqrt{ }$ & $\sqrt{ }$ & $\sqrt{ }$ & Present & 145 & $\mathrm{Zn}$ \\
\hline 31 & GmGLY|-16.1 & $\sqrt{ }$ & $\sqrt{ }$ & $\sqrt{ }$ & $\sqrt{ }$ & Present & 146 & $\mathrm{Zn}$ \\
\hline 32 & GmGLYI-16.2 & $\sqrt{ }$ & $\sqrt{ }$ & $\sqrt{ }$ & $\sqrt{ }$ & Present & 145 & $\mathrm{Zn}$ \\
\hline 33 & GmGLYI-16.3 & $\sqrt{ }$ & $\sqrt{ }$ & - & - & Absent & 96 & - \\
\hline 34 & GmGLYI-17.1 & $\sqrt{ }$ & - & $\sqrt{ }$ & $\sqrt{ }$ & Absent & 119 & - \\
\hline 35 & GmGLYI-18.1 & $\sqrt{ }$ & - & $\sqrt{ }$ & $\sqrt{ }$ & Absent & 117 & - \\
\hline 36 & GmGLYI-19.1 & $\sqrt{ }$ & - & $\sqrt{ }$ & $\sqrt{ }$ & Absent & 121 & - \\
\hline 37 & GmGLYI-20.1 & $\sqrt{ }$ & - & $\sqrt{ }$ & $\sqrt{ }$ & Absent & 121 & - \\
\hline 38 & GmGLYI-21.1 & $\sqrt{ }$ & $\sqrt{ }$ & $\sqrt{ }$ & $\sqrt{ }$ & Present & 122 & $\mathrm{Ni}$ \\
\hline 39 & GmGLYI-22.1 & $\sqrt{ }$ & - & $\sqrt{ }$ & $\sqrt{ }$ & Absent & 119 & - \\
\hline 40 & GmGLYI-23.1 & $\sqrt{ }$ & - & $\sqrt{ }$ & $\sqrt{ }$ & Absent & 117 & - \\
\hline 41 & GmGLYI-24.1 & $\sqrt{ }$ & - & $\sqrt{ }$ & - & Absent & 126 & - \\
\hline
\end{tabular}




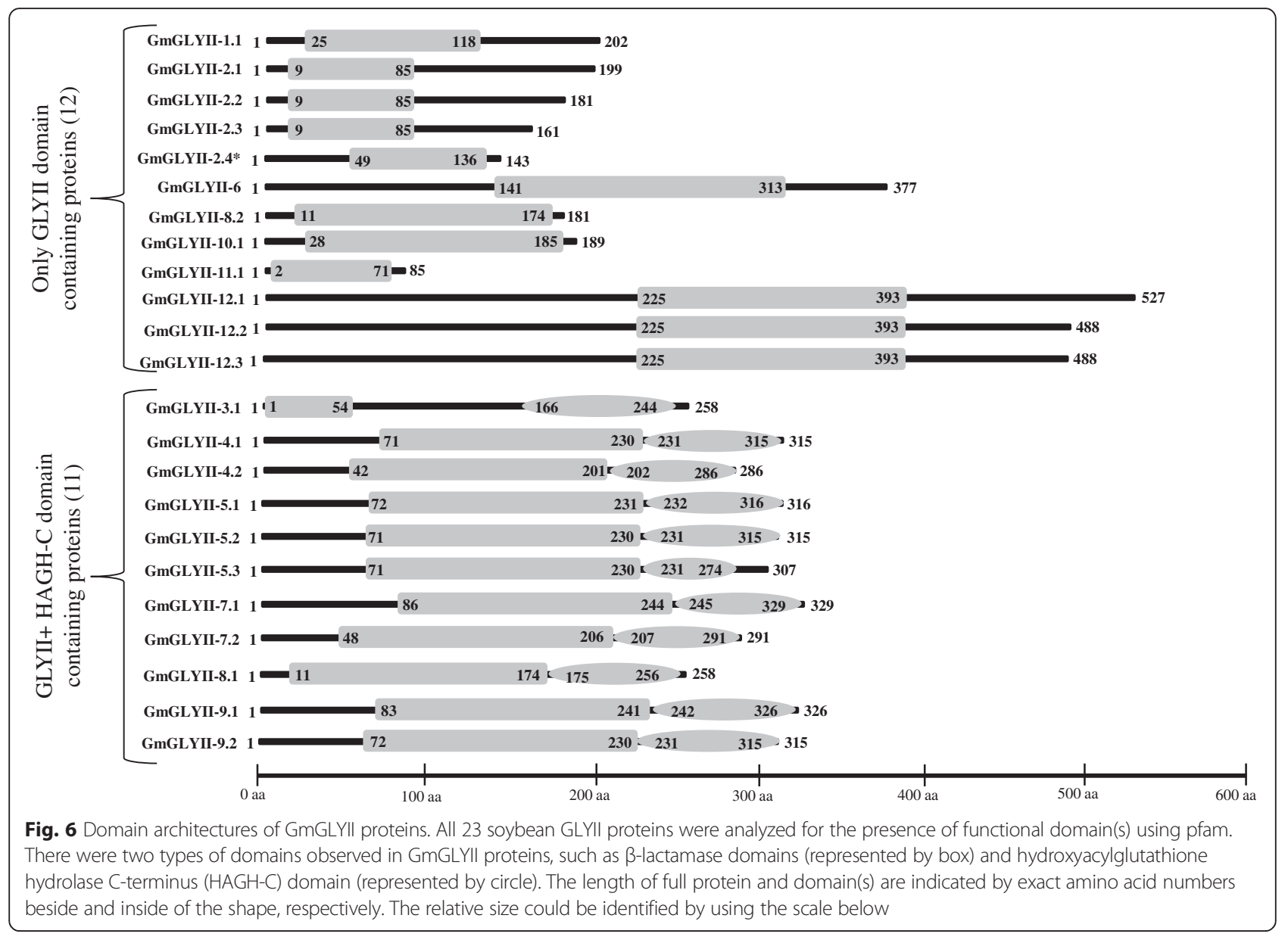

GmGLYII proteins, their protein sequences were aligned by multiple sequence alignment (Fig. 7). Both these motifs were indicated by black boxes (Fig. 7); their presence and absence were listed in Table 5 . Out of 23 putative GmGLYII proteins only three of them do not possess the conserved metal binding residues, but all of them have the active site motif (Fig. 7 and Table 5). Thus, it could be expected that all the predicted GmGLYII proteins have the functional GLYII enzyme activity except GmGLYII-1.1, GmGLYII-2.4, and GmGLYII-11.1 (Table 5).

\section{Homology modelling of representative GmGLYI and GmGLYII members}

To know the arrangement of active site residues and overall 3-D coordination, homology model of GmGLYI-3, GmGLYI-16 and GmGLYII-5 proteins was built (Fig. 8) based on the closely related template structure of Zea mays GLYI (PDB: 5D7Z) [43], mouse GLYI (PDB: 4OPN), and AtGLYII-2 (PDB: 2Q42) [30] proteins, respectively. GmGLYI-3 is a $\mathrm{Ni}^{2+}$-dependent monomeric GLYI enzyme (Fig. 8a), while GmGLYI-16 is a $\mathrm{Zn}^{2+}$-dependent homodimeric enzyme (Fig. 8b).
GmGLYI-3 has two putative active sites; one consists of H-156, E-204, Q-217 and E-268, and the other one consists of H-87, E-138, Q-286 and A-334. The second putative active site has lack of a highly conserved Glu residues, thus might be inactive in nature like previously reported OsGLYI-11.2 [12]. The $\mathrm{Zn}^{2+}$-dependent GmGLYI-16 consists of single GLYI domain (Fig. 4) and thus forms homo-dimer to create two putative active sites (Fig. 8b). One putative active site has Q80 and E146 (from one chain, A) and H174 and E220 (from another chain, B); another one has opposite members from both chains. Here both the active sites have all four conserved residues and thus are predicted to be functionally active too. On the other hand, GmGLYII-5 is a monomeric protein consists of two structural orientations, an N-terminal domain (L63 to D193) with two $\beta \beta \beta \alpha \beta$ topology and a C-terminal domain (T194 to F316) with five $\alpha$ helices (Fig. 8c). The metal binding and active site residues are Asn116, His118, Asp120, His121, His174, Asp193 and His231 (Fig. 8c) are found to be conserved as compared to template AtGLYII-2 protein. 


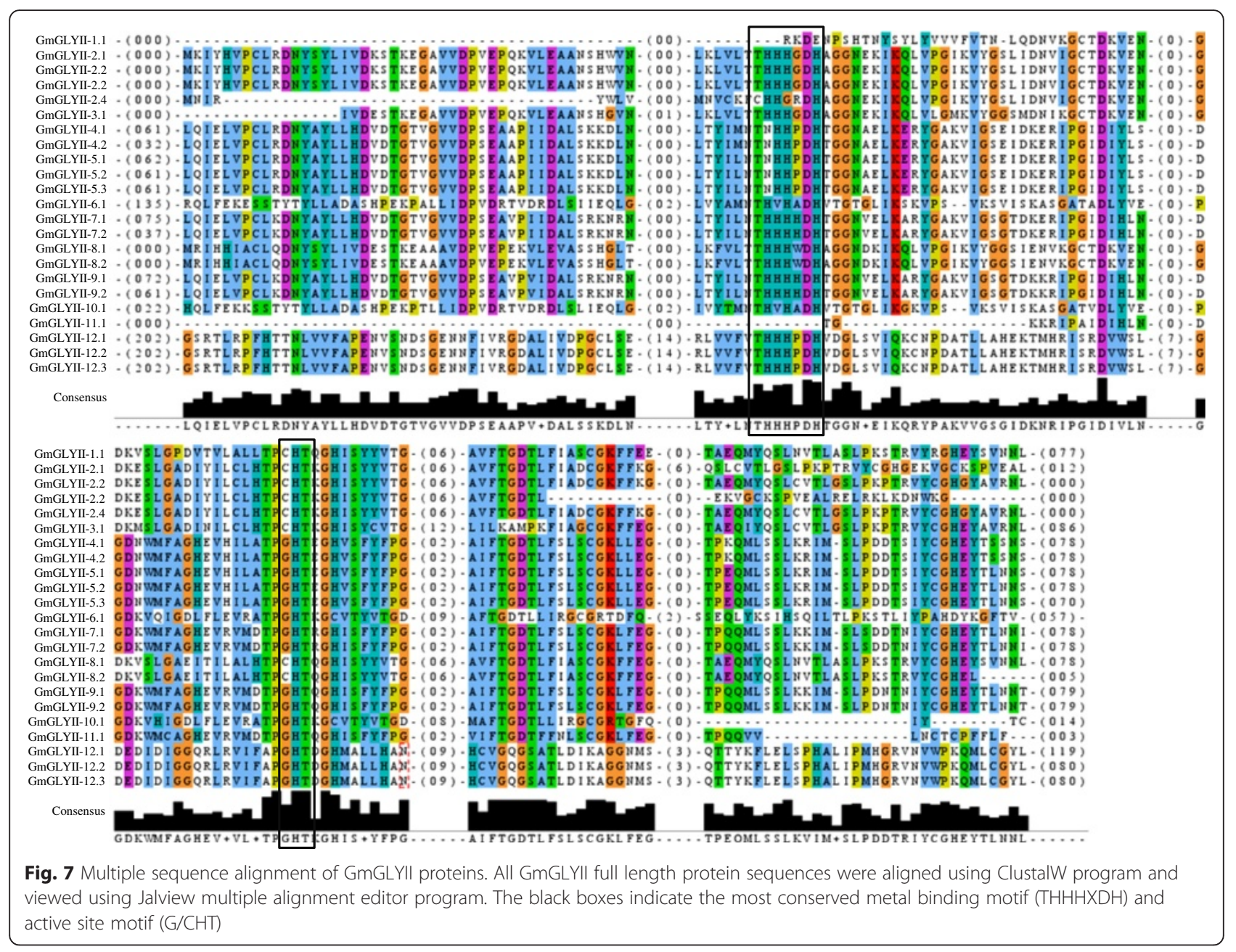

Expression analysis of GmGLYI and GmGLYII genes at different soybean tissues

RNA-Seq Atlas of Glycine max provides high-resolution gene expression data in a diverse set of 14 soybean tissues such as young leaf, flower, one $\mathrm{cm}$ pod (7 days after flowering, DAF), pod-shell(10 DAF and 14 DAF), seed $(10,14,21,25,28$ and 35 DAF), root and nodule. All these tissues could be broadly divided into three classes; such as underground, aerial and seed. RNA-seq normalized expression data for all GmGLYI and GmGLYII genes were retrieved from soybase (http://www.soybase.org/ soyseq/), except GmGLYI-14 and GmGLYI-15 due to lack of their appropriate probe (Additional file 2: Table S5). Data were analyzed and represented as heat maps generated using TIGR MeV software package (Fig. 9a and b). Expression analyses of all GmGLYI genes revealed that the different members have different tissue specific expression. Among all the 22 analyzed genes, GmGLYI-7 showed highest level of constitutive expression in all the tissues, followed by GmGLYI-21, GmGLYI-10 and GmGLYI-6. This high level of constitutive expression indicates their significant role at all these plant tissues (Fig. 9a). A cluster of genes showed medium to high level of expression in all the underground and aerial tissues only, followed by very low expression at the seed tissues. They are GmGLYI-2, GmGLYI-13, GmGLYI-17, GmGLYI-8, GmGLYI-4 and GmGLYI-16. Previous studies on rice and Arabidopsis showed the presence of highly seed specific GLYI genes such as AtGLYI-8, OsGLYI-3 and OsGLYI-10 [12]. Similarly, three of GmGLYI genes such as GmGLYI-1, GmGLYI-11 and GmGLYI-22 showed medium level of expression in different seed tissues only (Fig. 9a), indicating the evolutionary conservance for the presence of seed specific GLYI genes.

Expression analyses of GmGLYII genes indicate two clear clades (Fig. 9b). Out of 12 analyzed genes, five genes such as GmGLYII-1, GmGLYII-2, GmGLYII-3, GmGLYII-10 and GmGLYII-11 showed almost undetectable expression in all the tissues with few exceptions. Among others, GmGLYII-8 showed highest level of constitutive expression in all the tissues, followed by GmGLYII-6. These two genes might play a major role in all tissues. Similar to GmGLYI, a cluster of genes 
Table 5 Sequence analyses of all putative GmGLYII proteins for the presence of conserved motifs and enzyme activity

\begin{tabular}{|c|c|c|c|c|}
\hline SI. no & Proteins & Conserved metal binding motif (THHHXDH) & Active site motif (C/GHT) & Expected GLYII enzyme activity \\
\hline 1 & GmGLYII-1.1 & Absent & Present & No \\
\hline 2 & GmGLYII-2.1 & Present & Present & Yes \\
\hline 3 & GmGLYII-2.2 & Present & Present & Yes \\
\hline 4 & GmGLYII-2.3 & Present & Present & Yes \\
\hline 5 & GmGLYII-2.4 & Absent & Present & No \\
\hline 6 & GmGLYII-3.1 & Present & Present & Yes \\
\hline 7 & GmGLYII-4.1 & Present & Present & Yes \\
\hline 8 & GmGLYII-4.2 & Present & Present & Yes \\
\hline 9 & GmGLYII-5.1 & Present & Present & Yes \\
\hline 10 & GmGLYII-5.2 & Present & Present & Yes \\
\hline 11 & GmGLYII-5.3 & Present & Present & Yes \\
\hline 12 & GmGLYII-6.1 & Present & Present & Yes \\
\hline 13 & GmGLYII-7.1 & Present & Present & Yes \\
\hline 14 & GmGLYII-7.2 & Present & Present & Yes \\
\hline 15 & GmGLYII-8.1 & Present & Present & Yes \\
\hline 16 & GmGLYII-8.2 & Present & Present & Yes \\
\hline 17 & GmGLYII-9.1 & Present & Present & Yes \\
\hline 18 & GmGLYII-9.2 & Present & Present & Yes \\
\hline 19 & GmGLYII-10.1 & Present & Present & Yes \\
\hline 20 & GmGLYII-11.1 & Absent & Present & No \\
\hline 21 & GmGLYII-12.1 & Present & Present & Yes \\
\hline 22 & GmGLYII-12.2 & Present & Present & Yes \\
\hline 23 & GmGLYII-12.3 & Present & Present & Yes \\
\hline
\end{tabular}

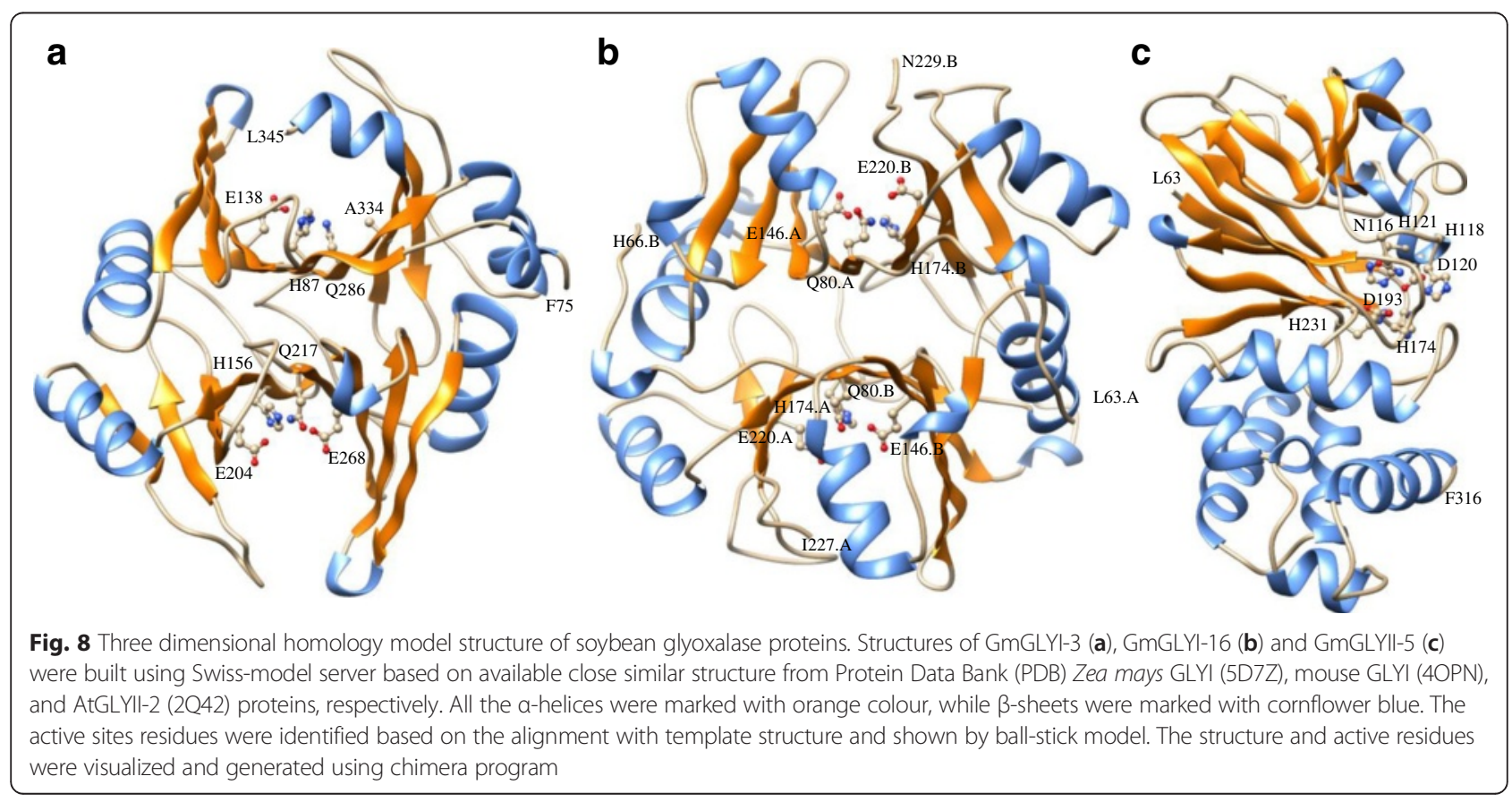




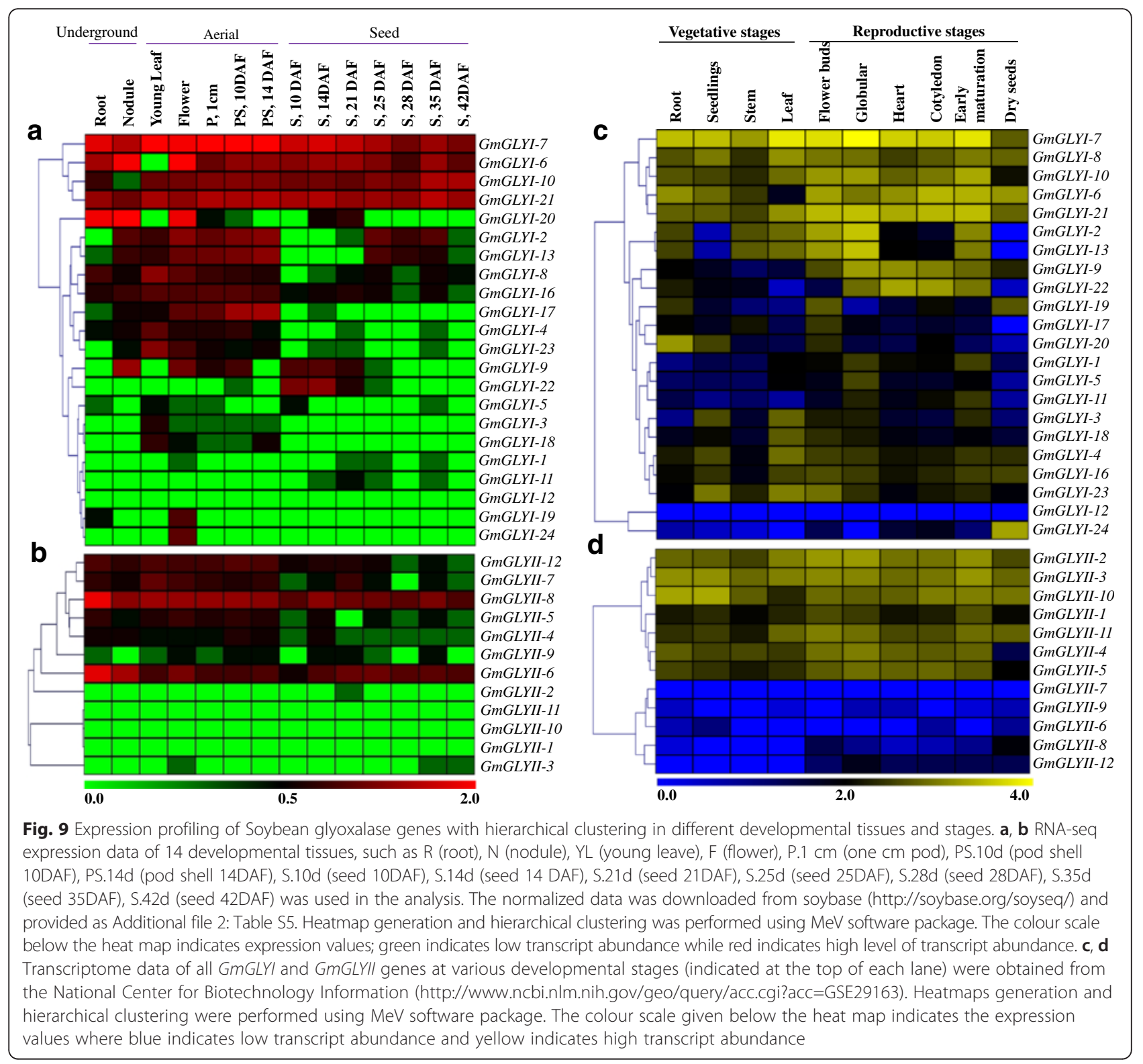

(GmGLYII-4, GmGLYII-5, GmGLYII-7, and GmGLYII12) showed medium level of expression in the underground and aerial tissues, except the seed (Fig. 9b). No tissue specific expression pattern was observed in case of GmGLYII genes.

From the expression data analysis of the identified paralogous pairs of GmGLYI and GmGLYII genes in 14 soybean tissues revealed a high level of expression divergence. For example, GmGLYI-6 showed high level constitutive expression while its paralogous GmGLYI-9 showed detectable expression in a few tissues. However, some of the paralogous GmGLYI gene pairs namely GmGLYI-1/-11, GmGLYI-2/-13, GmGLYI-4/-8, GmGLYI10/-21, and GmGLYI-19/-24 showed similar pattern of expression. The divergence is even more in case of GmGLYII gene pairs. For instance, GmGLYII-8 is highly expressed in all the analyzed tissues while its paralogous counterpart GmGLYII-1 remains mostly undetectable. Similar level of deviation was also observed in case of GmGLYII-6/-10and GmGLYII-7/-9 gene pairs.

\section{Expression analysis of GmGLYI and GmGLYII genes at different developmental stages}

Expression of GmGLYI and GmGLYII genes at different developmental stages was analyzed using publicly-available genome-wide transcript profiling data of soybean (http:// www.ncbi.nlm.nih.gov/geo/query/acc.cgi?acc=GSE29163). The dataset contains mainly two broad developmental sets, one at vegetative stages (roots, seedlings, stems, leaves) and the other at reproductive stage (floral buds, 
different stages of seed development- globular, heart, cotyledon, early-maturation, dry). As shown in Fig. 9c, most of the GmGLYI genes showed high level of expression, without any distinct pattern of expression. Out of the 24 GmGLYI genes, only GmGLYI-12 showed undetectable expression at all stages. Among others, GmGLYI-7 showed maximum constitutive expression in all the developmental stages followed by GmGLYI-21, GmGLYI-6, GmGLYI-10 and GmGLYI-8 (Fig. 9c). Two of GmGLYI members, GmGLYI-22 and GmGLYI-9 showed only reproductive stage specific expression. This indicates the development specific modulation of GmGLYI gene expression.

On the other hand, a distinct division is observed in the GmGLYII gene expression through the developmental stages (Fig. 9d). A cluster of genes such as GmGLYII1, GmGLYII-2, GmGLYII-3, GmGLYII-6, GmGLYII-10, and GmGLYII-11 showed either undetectable or very low level of expression in both vegetative and reproductive phases. However, rest of the GmGLYII members showed medium to high level of expression in all the developmental stages constitutively (Fig. 9d). High level of expression of both GmGLYI and GmGLYII genes in all the developmental stages of soybean indicates the constitutive metabolic/cellular role of glyoxalase pathway throughout the life cycle of plant.

\section{Expression analysis of soybean glyoxalase genes under} stress

To gain deep insights into the function of glyoxalase genes in the abiotic stress adaptation of soybean, the expression profiles of all GmGLYI and GmGLYII genes were analyzed in response to salinity and drought stresses using publicly available microarray data. Expression data sets were retrieved from gene expression omnibus database of GSE41125 and GSE40627 for salinity and drought stress, respectively. Data were not available for all these genes due to the limitation of respective probes. Among the total of 24 GmGLYI and 12 GmGLYII genes; data for 19 GmGLYI and eight GmGLYII genes were analyzed for salinity, while expression data of 21 GmGLYI and 12 GmGLYII genes were found for drought stress. Different glyoxalase members responded differentially in terms of their expression towards both these stresses (Fig. 10a and b). In response to salinity stress, four GmGLYI genes and two GmGLYII genes showed up-regulation, while seven GmGLYI genes and four GmGLYII genes showed down-regulation and rest of them remained unaltered (Fig. 10a and b). Similarly, drought stress causes upregulation of seven GmGLYI genes and five GmGLYII genes, and downregulation of eight GmGLYI genes and six GmGLYII

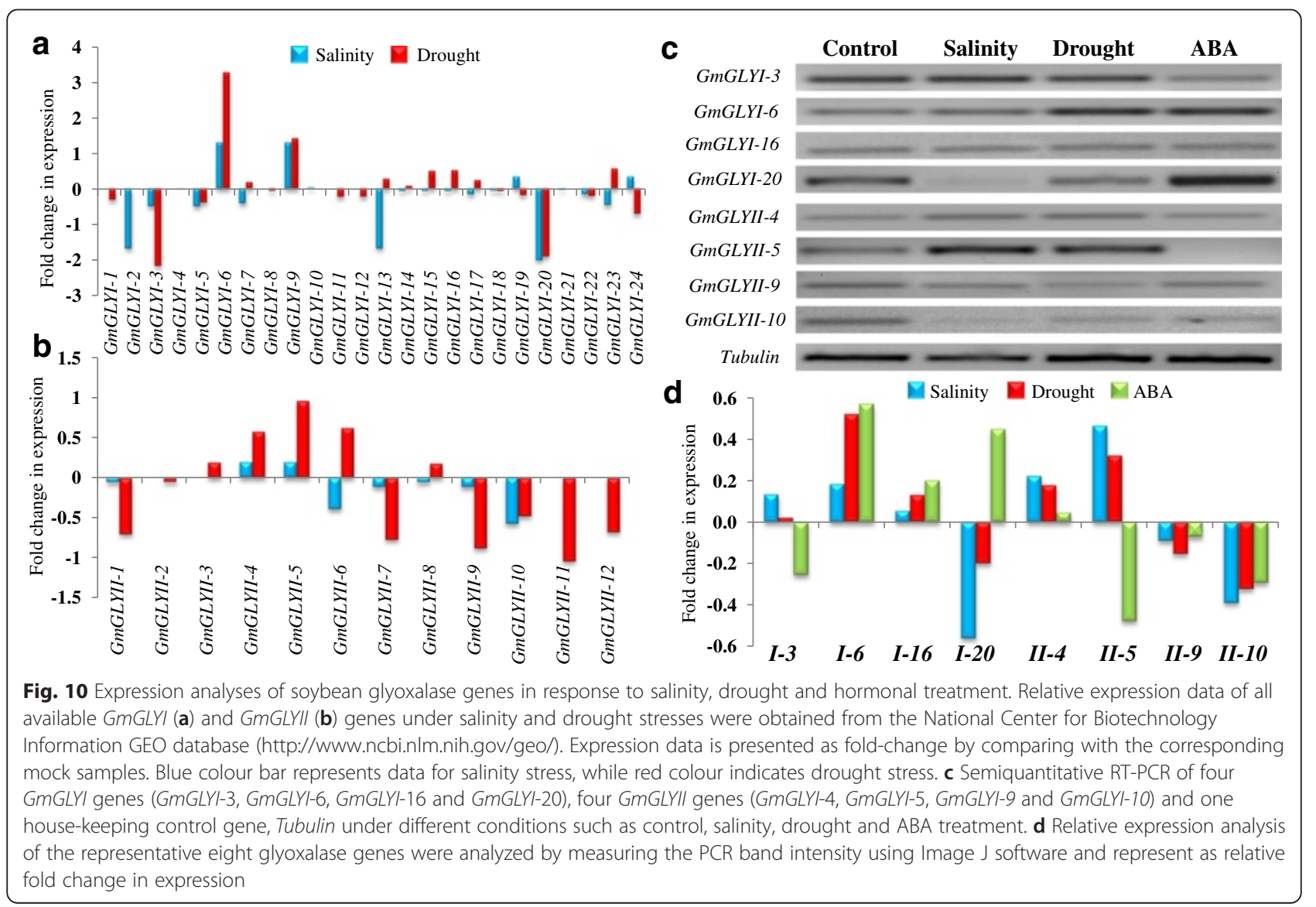


genes (Fig. 10a and b). Among 24 GmGLYI genes; GmGLYI-6 and GmGLYI-9 showed significant upregulation, while other two of them (GmGLYI-3 and GmGLYI-10) showed down-regulation in both the stresses (Fig. 10a). In case of GmGLYII; GmGLYII-4 and GmGLYII-5 showed up-regulation, while GmGLYII-10 showed significant down-regulation in both salinity and drought stresses (Fig. 10b). Rest of the members of both GmGLYI and GmGLYII family showed variable expression pattern. This indicates diverse role of different glyoxalase members in the stress modulation pathways of soybean plant.

To screen the role of soybean glyoxalase genes in response to salinity, drought and hormonal treatment (Abscisic Acid, ABA), a semi quantitative RT-PCR was performed to validate four candidate GmGLYI genes (GmGLYI-3, -6,-16 and -20) and four candidate GmGLYII genes (GmGLYII-4, -5,-9 and -10) which were highly responsive in microarray data analysis (Fig. 10a and b). For this purpose, 15 days old soybean seedlings were subjected to normal water (as control), $200 \mathrm{mM}$ $\mathrm{NaCl}$ (for salinity) or withdrawn of water (for drought) or $10 \mathrm{mM} \mathrm{ABA}$ (for hormonal treatment) for $8 \mathrm{~h}$. Expression of all the candidate genes were compared with that of Tubulin (act as a house-keeping control gene) (Fig. 10c). Relative transcript abundance of all these transcripts was measured by scanning the gel image using Image J software relative fold change in expression was calculated considering Tubulin as internal control (Fig. 10d). It could be clearly inferred from Fig. 10d that GmGLYI-6, GmGLYII-4 and GmGLYII-5 showed strong up-regulation in response to both salinity and drought, while GmGLYI-20, GmGLYII-9 and GmGLYII-10 showed clear down-regulation (Fig. 10d). The remaining two members, GmGLYI-3 and GmGLYI-16 showed slight up/ down regulation as compared to control sample. Overall, the pattern of expression of these eight candidate genes (Fig. 10c) was found to be almost similar to that of microarray data (Fig. 10a and b).

\section{Identification of cis-elements in the promoter region of soybean glyoxalase genes}

In order to comment on stress responsive expression of GmGLY genes in response to salinity, drought and ABA treatment, $1 \mathrm{~kb}$ upstream promoter region of each GmGLYI and GmGLYII genes were retrieved from soybase (http://www.soybase.org/dlpages/flank/index.php) and analyzed for the presence of cis-acting elements using PlantCARE [44]. This analysis leads to the identification of several stress-responsive cis elements such as abscisic acid responsive element (ABRE), auxin responsive element (AuxRR-core), fungal elicitor responsive element (BOX-W1), ethylene responsive element (ERE), gibberellin-responsive element (GARE), heat shock element (HSE), jasmonate and elicitor responsive element (JERE), low temperature responsive element (LTR), MYB-binding site (MBS), defence and stress responsive element (TC-rich), wounding and pathogen responsive elements (W-box and WUN-motif), salicylic acid responsive element (TCA), Methyl jasmonate-responsive element (CGTCA box and TGACG motif), element conferring high transcription level (5' UTR Py-rich stretch). All these motifs are very crucial for plant stress modulation pathways and thus play important role to regulate the expression of various stress responsive genes $[45,46]$. All these motifs were found to be distributed randomly in both the positive and negative strands of promoter sequences (Fig. 11). Among GmGLYI members, GmGLYI-1 and GmGLYI-24 have maximum cis-elements (12 elements), while GmGLYI14 promoter has minimum two elements on it. In case of GmGLYII members, GmGLYII-7 has maximum ten elements, while GmGLYII-8 has only one element. ABRE, HSE, and TGACG motif are found to be present in almost every promoter of GmGLY genes with few exceptions. Although correlation between the presence of cis-acting regulatory elements and the observed transcript abundance needs to be confirmed experimentally, these results indicated the stress-responsive nature of GmGLY genes.

\section{Discussion}

Methylglyoxal (MG) is a metabolic by-product generated naturally in all living cells [7]. But the level of MG goes up in response to various abiotic stresses in plants [9]. It has been established in literature that glyoxalase pathway plays a vital role in the detoxification of MG as well as provides tolerance against multiple abiotic stresses $[5,7]$. Genome wide analysis of glyoxalase pathway has been done preliminary in the monocot model plant rice and dicot model plant Arabidopsis [1]. However, this family has not been studied in any other species including legume. In the present study, we have performed a genome wide analysis of soybean to identify glyoxalase gene families, including their chromosomal location, gene and protein structure, conserved active site and catalytic site motifs and expression profiles. A total of 24 GLYI and 12 GLYII genes were identified in the soybean genome that codes for 41 GLYI and 24 GLYII proteins, respectively (Tables 1 and 2). The number of GmGLYI genes is 2.2 times more than that of Arabidopsis and rice (eleven genes each); and GmGLYII shows 2.4 times more abundance than that of Arabidopsis (five AtGLYII genes), and 4 times more abundant than rice (three OsGLYII genes). The possible reason behind this significant increase in gene number might be the two duplication events of soybean [14] that has occurred after the monocot/dicot split, or most of the soybean genes expanded in a species-specific manner [17]. 


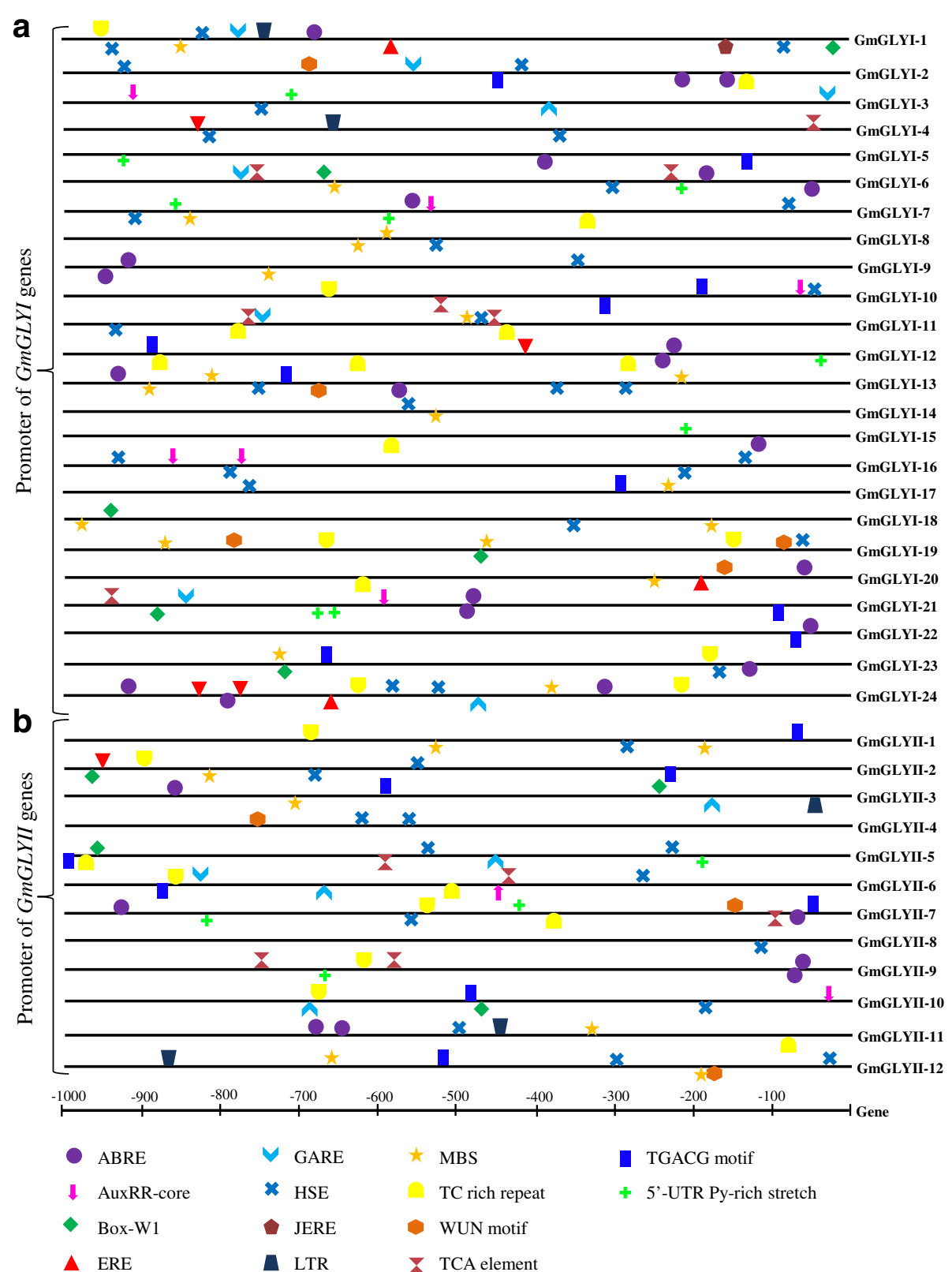

Fig. 11 In silico promoter analysis of GmGLYI and GmGLYIl genes. One kb 5' upstream sequence of all GmGLYI and GmGLYIl genes was downloaded from soybase database and scanned through PlantCARE for the identification of number and position of various cis-acting regulatory elements. Different regulatory elements were indicated by different colour symbols and placed in their relative position on the promoter. Symbols presented above the line indicate forward strand of DNA, while below one indicates the reverse strand

To adopt with different adverse environmental conditions, plants tend to duplicate genes to generate novel members or increase number $[17,47]$. There are three basic principal patterns of gene duplications, such as tandem duplication, segmental duplication and transposition. In the present analysis, a total of ten duplicated pairs were observed in GmGLYI family and five in GmGLYII family (Table 3). All of them showed segmental duplication except one, that is the major pattern of gene duplication in plant. The tandem duplication pair
GmGLYI-14/-15 was formed 33.9 Mya ago; while the segmental duplications of GmGLYI genes have occurred between 3.7 and 13.6 Mya and that of GmGLYII occurred between 6.3 and18.8 Mya. This indicates that the tandem duplication event has occurred before the segmental duplication event. Similar pattern of duplication has been reported previously for HD-Zip genes of soybean [17].

Soybean, including other plants has been found to possess greater number of GLYI and GLYII genes and 
proteins as compared to their animal counterpart to date. One of the possible reasons behind this is gene duplication of plant during evolution that ultimately leads to functional divergence of genes [48]. Functional divergence might lead to either subfunctionalization or neofunctionalization, that in turn resulted in novel gene functions [48]. In the present study, out of 41 predicted GLYI proteins only twenty of them possess all four conserved metal binding sites and are expected to have functional GLYI enzyme activity (Fig. 5 and Table 4). The other proteins might be functionally diverged and possess some other activities similar to GLYI. Structurally GLYI is the member of vicinal oxygen chelate (VOC) superfamily that includes extradiol dioxygenases, GLYI and methylmalonyl-CoA epimerase [49]. One of the earlier predicted GmGLYI (Accession no. X68819) was found to have Glutathione S-transferase activity, too [50]. Apart from that, it has been wellcharacterized in literature that there are two metal activation classes of GLYIs; $\mathrm{Zn}^{2+}$ and non- $\mathrm{Zn}^{2+}$ (mainly $\left.\mathrm{Ni}^{2+} / \mathrm{Co}^{2+}\right)$. Both these classes possess same four conserved metal binding residues and octahedral metal co-ordination; regardless of the metal activation class [51]. Three dimensional structure of one of the predicted $\mathrm{Ni}^{2+}$-dependent GLYI, GmGLYI-3 (Fig. 8a) and $\mathrm{Zn}^{2+}$-dependent GLYI, GmGLYI-16 (Fig. 8b) confirms the presence of same active site residues in both. However, $\mathrm{Ni}^{2+}$-dependent GmGLYI-3 found to be monomer consisting of two GLYI domains that fold to create two putative active sites (Fig. 8a). Whereas, single GLYI domain containing $\mathrm{Zn}^{2+}$-dependent GmGLYI-16 need to be homo- dimer to create two putative active sites (Fig. 8b). Interestingly, metal specificity of putative GLYIs could be easily predicted based on the protein's amino acid length and sequence $[2,51] . \mathrm{Zn}^{2+}$ - activated GLYIs are relatively larger in amino acid length than $\mathrm{Ni}^{2+} / \mathrm{Co}^{2+}$-activated ones and have unique region in their sequence (Fig. 5). Based on these criteria, 16 out of 20 predicted functional GmGLYI enzymes are expected be $\mathrm{Ni}^{2+} / \mathrm{Co}^{2+}$-activated. Same pattern of dominance by $\mathrm{Ni}^{2+} / \mathrm{Co}^{2+}$-activated forms was observed in case of rice GLYIs (3 out of 4 expected active OsGLYIs) and Arabidopsis GLYIs (two out of three expected functional AtGLYIs) [2].

On contrary, GLYII enzymes contain the $\beta$-lactamase fold structure that includes lactonase, rubredoxin:oxygen oxidoreductase, GLYII, arylsulfatase, phosphodiesterase, carboxylesterase and tRNA maturase [52]. Previously, GLYII family members from Arabidopsis (AtGlx2-5) and rice (OsGLYII-1) have been reported to lack of GLYII activity, instead they possess sulphur dioxygenase like ethylmalonic encephalopathy protein 1 (ETHE1) activity [11]. Similar to GLYI, three out of 23 predicted GmGLYII proteins did not possess the conserved metal binding motif that might have resulted in the absence of GLYII activity and leads to the functional divergence.

Expression of glyoxalase genes has been found to be highly specific towards certain tissue or developmental stages in Arabidopsis and rice [1]. Thus, the expression pattern of GmGLYI and GmGLYI genes was analyzed at different developmental stages and tissues (Fig. 9). These data revealed the tissue specific expression pattern of glyoxalase genes in soybean too. Out of all, GmGLYI-7, GmGLYI-21 and GmGLYII-8 are found to be the constitutively expressed members of soybean glyoxalase system. A cluster of GmGLYI and GmGLYII genes maintained high level of expression in all the underground and aerial tissues, followed by low level of expression in the different stages of seed development (Fig. 9). This indicates the presence of functional distribution among the multiple members in different tissue/developmental stimuli. On contrary, some of the GmGLYI genes such as GmGLYI-1, GmGLYI-11, and GmGLYI-22 showed medium level of expression in the seed tissues only, with low or no expression in other parts (Fig. 9). This indicates development specific transition/regulation of GmGLYI genes. In case of GmGLYII genes, two distinguishable clades were observed in their expression where one set showed high level expression in all the tissues and another have no expression at all. These low expressive genes might have other cellular/metabolic regulation expect developmental/tissue regulation. Another interesting expression pattern was observed for soybean glyoxalase genes under abiotic stresses (Fig. 10). Different members of GmGLYI and GmGLYII families responded differentially against salinity, drought and hormone (ABA) treatment (Fig. 10c). GmGLYII-9 and GmGLYII-10 showed strong down-regulation in response to all three conditions, while GmGLYII-16 showed sharp up-regulation (Fig. 10d). Presence of various cis-acting regulatory elements on the putative promoter sequence of GmGLYI and GmGLYII genes might be a probable reason behind this altered expression (Fig. 11). Similar pattern of expression was observed previously in rice and Arabidopsis glyoxalase genes [1], where each member shows specific pattern of expression towards the particular type of stress treatment. Overall, the observed information in the present study will facilitate to find out the appropriate candidate gene(s) for further functional characterization and raising stress-tolerant transgenic crop plants.

\section{Conclusions}

Taken together, we have performed a comprehensive in silico analysis of soybean glyoxalase gene families (GmGLYI and GmGLYII), and provided detailed information about them. Specifically, our results show that soybean genome contains 24 GmGLYI and 12 GmGLYII 
genes that code for 41 GmGLYI and twenty-three GmGLYII proteins, respectively; the largest identified glyoxalase gene family to date in any species/organism. Present study indicates genome-wide duplication (both segmental and tandem) of glyoxalase genes that lead to the expansion of family. Based on the presence of conserved motifs and sequence homology, we have provided insight into their putative function and metal dependency. Finally, expression data confirms the development, tissue and stress specific response of each and every gene in spite of having large multi-member family.

\section{Methods}

\section{Identification of GmGLYI and GmGLYII genes in soybean}

The putative GLYI and GLYII proteins in soybean genome were identified by BLASTP search against the new soybean genome database (Wm82.a2.v1) (http://www.soybase.org/) [53] with an e-value of 1 using previously reported soybean GLYI protein sequence (GenBank: NM_001249223.1) and Brassica juncea GLYII protein sequence (GenBank: AAO26580.1) as query, respectively. Subsequently, each of the identified sequences was used as secondary queries to find other new members. All the protein sequences were checked individually using Pfam (http://pfam.xfam.org/) with default parameters and e-value of 1 , for the presence of glyoxalase domain (PF00903) in GLYI proteins and metallo-beta-lactamase domain (PF00753) in the GLYII proteins. All the identified putative glyoxalase proteins were nomenclature as prefix "Gm" for Glycine max, followed by GLYI or GLYII and Arabic numbers serially starting from 1 depending on their chromosomal position. Alternate splice forms were represented by adding arabic numbers after "." sign sequentially. The chromosomal locations for all the putative GmGLYI and GmGLYII genes were identified from soybase (http://soybase. org/gb2/gbrowse/gmax1.01/) [53] database to draw the chromosomal map. Various physio-chemical properties of all the identified GmGLYI and GmGLYII proteins were calculated using Prot-Param software (http://web.expasy.org/protparam/). Localization of proteins were predicted using CELLO v.2.5: sub-cellular localization predictor (http://cello.life.nctu.edu.tw/) [23] and pSORT prediction software (http://www.genscript. com/wolf-psort.html) [24]. Chloroplast localization was further confirmed by ChloroP (http://www.cbs.dtu.dk/ services/ChloroP/) [25].

\section{Multiple sequence alignment and phylogenetic analysis}

To investigate the phylogenetic relationship and conserved motifs/metal binding sites among GLYI and GLYII proteins from various plant species, sequences were downloaded from NCBI (http://www.ncbi.nlm.nih. gov/), PDB (http://www.rcsb.org/pdb/home/home.do), rice genome database (http://rice.plantbiology.msu.edu/),
Arabidopsis genome database (https://www.arabidopsis.org/) and soybean database (http://www.soybase.org/). Protein sequences used in the study of phylogenetic analysis were available in Additional files 4 and 5. Multiple sequence alignment was performed using ClustalW [54] and phylogenetic tree was constructed using MEGA 5.2 [55] with Neighbour-Joining method and 1000 bootstrap replicates.

\section{Gene duplication and $\mathrm{Ka} / \mathrm{Ks}$ calculation}

Gene duplication was analyzed using plant genome duplication database (http://chibba.agtec.uga.edu/duplication/ index/downloads) [26] for soybean. Genes having more than $90 \%$ sequence similarities were considered as segmental duplication, while tandem duplicated genes were separated by five or fewer genes in a 100-kb region. Synonymous (Ks) and nonsynonymous substitution (Ka) rates were retrieved from plant genome duplication database or calculated from PAL2NAL program (http://www.bork. embl.de/pal2nal/) [56]. Divergence time (in millions of years) was calculated for each gene pair considering a rate of $6.1 \times 10^{-9}$ substitutions per site per year [17]. Thus, divergence time $(\mathrm{T})=\mathrm{Ks} /\left(2 \mathrm{X} 6.1 \times 10^{-9}\right) \mathrm{X}^{-6} 0^{-6}$ Mya.

\section{Assessment of domain architecture, catalytic conservance and metal ion specificity of GLYI and GLYII proteins}

All the predicted GmGLYI (41) and GmGLYII (24) proteins were analyzed using Pfam to reveal the presence of conserved glyoxalase domain (PF00903) and metallo-betalactamase domain (PF00753), respectively. Glyoxalase domain (PF00903) of GmGLYI and metallo-beta-lactamase domain (PF00753) of GmGLYII proteins were aligned separately with previously characterized members using ClustalW and analyzed for the presence of conserved motifs. GLYI has a conserved H/QEH/QE motif for metal binding and catalysis, whereas GLYII has two separate metal binding motif (THXHXDH) and active site motif (C/GHT). The metal ion specificity of GmGLYI proteins was predicted based on the previous studies [2, 51].

\section{Homology based structural modelling of various soybean glyoxalase proteins}

Homology based model of GmGLYI-3, GmGLYI-16 and GmGLYII-5 was built using SWISSMODEL program (http://swissmodel.expasy.org/) [57]. Respective protein sequences were first analyzed by template search, followed by model building using best template structure with highest similarities. Structures of GmGLYI-3, GmGLYI-16 and GmGLYII-5 were built using most similar structure available from Protein Data Bank (PDB) i.e. Zea mays GLYI (5D7Z), mouse GLYI (4OPN), and AtGLYII-2 (2Q42) proteins, respectively. Resulting structures were visualized using UCSF Chimera (http://www.cgl.ucsf.edu/ 
chimera) [58]. Active site residues were identified and marked based on previous template structure analysis.

\section{Expression analysis using RNA-Seq Atlas of Glycine max} To analyze the tissue-specific expression data of 24 GmGLYI and 12 GmGLYII genes, their corresponding probe sets were indentified using soybase tool (http:// www.soybase.org/correspondence/index.php). Normalized transcript data was downloaded from soybase (http://soybase.org/soyseq/) for 14 different tissues, including root, nodule (underground tissues); leaf, flower, pod-shell 10-day after flowering (DAF), pod-shell 14-DAF, one-cm pod (aerial tissues); and different stages of seed development (seed of 10-DAF, 14-DAF, 21-DAF, 25-DAF, 28-DAF, 35-DAF and 42-DAF). This normalized expression was used to generate heatmap and hierarchical clustering using the Institute for Genomic Research MeV software package [59].

\section{Expression analysis of GmGLYI and GmGLYII genes at different developmental stages}

Expression patterns of GmGLYI and GmGLYII genes at different developmental stages were determined using the publically available transcriptomes data (http://www.ncbi. nlm.nih.gov/geo/query/acc.cgi?acc=GSE29163). Transcript data of ten different soybean stages (root, seedlings, stem, leaf, flower buds, and different stages of seed development- globular, heart, cotyledon, early maturation, and dry seeds) were downloaded from the NCBI database (http://www.ncbi.nlm.nih.gov/) with accession numbers of SRX062325 to SRX062334. After normalization, the values were used for heatmap generation using the Institute for Genomic Research MeV software package [59].

\section{Expression analysis of GmGLYI and GmGLYII genes in response to salinity and drought stresses}

Expression data of glyoxalase genes in response to salinity and drought stresses were retrieved from the National Center for Biotechnology Information Gene Expression Omnibus (GEO) database [60] with accession numbers GSE41125 and GSE40627, respectively. Corresponding probe sets for GmGLYI and GmGLYII genes were identified using NetAffx Analysis Center (http://www.affymetrix.com/analysis/index.affx?navMo$\mathrm{de}=\mathrm{cat} 530006 \& \mathrm{aId}=$ netaff $\mathrm{xNav}$ ) online Probe Match tool. More than one gene with the same probe set, were considered as same transcriptional profile, while in case of gene having more than one probe set, the highest value was considered. Expression data were normalized using that of mock, and represented as bar diagram.

\section{Plant material, stress treatments and semiquantitative RT-PCR}

Soybean (Glycine max L. variety Sohag) seedlings were grown in a condition with continuous $30{ }^{\circ} \mathrm{C}$ temperature and $12 \mathrm{~h} / 12 \mathrm{~h}$ of photoperiod [17]. Fifteen days old seedlings were irrigated with normal water as experimental control or $200 \mathrm{mM} \mathrm{NaCl}$ solution for salinity stress or $10 \mathrm{mM} \mathrm{ABA}$ solution for hormonal treatment for $8 \mathrm{~h}$. Seedlings were placed onto filter paper and exposed to the air to mimic drought stress. Leaves were collected from all these seedlings after $8 \mathrm{~h}$ (with triplicates) and total RNA was extracted using TRIzol ${ }^{\circ}$ Reagent (Thermo Fisher Scientific, USA). First-strand cDNA was synthesized using RevertAid First Strand cDNA Synthesis Kit (Thermo Fisher Scientific, USA). Gene-specific primer for eight candidates genes, listed in Additional file 3: Table S6, were designed using Primer-Blast (http:// www.ncbi.nlm.nih.gov/tools/primer-blast/), and soybean Tubulin gene was used as an internal control [22].

\section{Promoter sequence analysis for putative cis-regulatory elements}

To identify various cis-acting regulatory elements in the promoter sequences of GmGLYI and GmGLYII genes, $1 \mathrm{~kb} 5^{\prime}$ upstream region sequences were retrieved from soybean genome database (http://www.soybase.org/ dlpages/flank/index.php). Promoter sequences were analyzed using PlantCARE databases [44] to find out the presence of cis-acting regulatory elements.

\section{Availability of data and materials}

All sequence information regarding soybean is available at a public database, Soybase (http://soybase.org/). Apart from that, most datasets supporting the conclusions of this article are included as additional files. All protein sequences used in the phylogenetic analysis had been already deposited in Uniprot (http://www.uniprot.org/) and provided as additional data too. The seeds of Soybean (Glycine $\max L$. variety Sohag) are available from Bangladesh Agriculture Research Institute, Gazipur, Bangladesh.

\section{Additional files}

Additional file 1: Figure S1. Phylogenetic relationship of GmGLYI (A) and GmGLYII (B) proteins. An unrooted tree was generated using Neighbor-Joining method with 1000 bootstrap by MEGA5.2 software using the full-length amino acid sequences of the twenty-four GmGLYI and twelve GmGLYII proteins (only the first splice variants were taken in case of multiple members). The numbers next to the branch shows the result of 1000 bootstrap replicates expressed in percentage, and scores higher than $50 \%$ are indicated on the nodes. (PDF 17 kb)

Additional file 2: Table S1. Pairwise identities between paralogous pairs of GLYI proteins from Soybean. Table S2. Percentage of identities between all GLYII proteins from Soybean. Table S5. Expression analysis of soybean GLYI and GLYII genes through RNA-seq data (XLS $40 \mathrm{~kb}$ )

Additional file 3: Table S3. Number of exons and introns in all the splice variants of GmGLYI genes. Table S4. Number of exons and introns in all the splice variants of GmGLYII genes. Table S6. Primers used in the semi-quantitative RT-PCR. (DOCX 26 kb) 
Additional file 4: Protein sequences used for phylogenetic analysis of GLYI. (DOCX 21 kb)

Additional file 5: Protein sequences used for phylogenetic analysis of GLYII. (DOCX 17 kb)

\section{Abbreviations}

aa: amino acid; ABA: abscisic acid; bp: base pair; DAF: day after flowering; GLYI: Glyoxalase I; GLYII: Glyoxalase II; GSH: reduced glutathione; h: hour; MG: methylglyoxal; Mya: million years.

\section{Competing interests}

The authors declare that they have no competing interest.

\section{Authors' contributions}

AG designed and performed the experiments, and analysed the data. TI performed semiquantitative RT-PCR experiment. AG and TI wrote the manuscript. Both authors read the manuscript and approved the final version.

\section{Acknowledgements}

AG acknowledges Shahjalal University of Science and Technology, Sylhet, Bangladesh for providing the logistic support and Department of Biochemistry and Molecular Biology of the same University for providing the laboratory space. TI acknowledges Plant Breeding and Biotechnology Laboratory, Department of Botany, Dhaka University for providing laboratory facilities. Authors thank Prof. Dr. M. Mozammel Haque, Department of Botany, University of Dhaka, Dhaka-1000, Bangladesh and Prof. Dr. Yasmeen Haque, Department of Physics, Shahjalal University of Science and Technology, Sylhet-3114, Bangladesh for their valuable time for copy-editing the manuscript.

\section{Author details}

${ }^{1}$ Department of Biochemistry and Molecular Biology, Shahjalal University of Science and Technology, Sylhet 3114, Bangladesh. ${ }^{2}$ Plant Breeding and Biotechnology Laboratory, Department of Botany, Dhaka University, Dhaka 1000, Bangladesh.

\section{Received: 8 September 2015 Accepted: 11 April 2016} Published online: 16 April 2016

\section{References}

1. Mustafiz A, Singh AK, Pareek A, Sopory SK, Singla-Pareek SL. Genomewide analysis of rice and Arabidopsis identifies two glyoxalase genes that are highly expressed in abiotic stresses. Funct Integr Genomics. 2011;11(2):293-305.

2. Kaur C, Vishnoi A, Ariyadasa TU, Bhattacharya A, Singla-Pareek SL, Sopory SK Episodes of horizontal gene-transfer and gene-fusion led to co-existence of different metal-ion specific glyoxalase I. Sci Rep. 2013:3:3076.

3. Rabbani N, Thornalley PJ. Glyoxalase in diabetes, obesity and related disorders. Semin Cell Dev Biol. 2011;22(3):309-17.

4. Thornalley PJ. The glyoxalase system: new developments towards functiona characterization of a metabolic pathway fundamental to biological life. Biochem J. 1990:269(1):1-11.

5. Kaur C, Ghosh A, Pareek A, Sopory SK, Singla-Pareek SL. Glyoxalases and stress tolerance in plants. Biochem Soc Trans. 2014; 42(2). doi:10.1042/ BST20130242.

6. Singla-Pareek SL, Reddy MK, Sopory SK. Genetic engineering of the glyoxalase pathway in tobacco leads to enhanced salinity tolerance. Proc Natl Acad Sci U S A. 2003:100(25):14672-7.

7. Kaur C, Singla-Pareek SL, Sopory SK. Glyoxalase and methylglyoxal as biomarkers for plant stress tolerance. Crit Rev Plant Sci. 2014;33(6):429-56.

8. Guo YL. Gene family evolution in green plants with emphasis on the origination and evolution of Arabidopsis thaliana genes. Plant J. 2013;73(6): 941-51.

9. Ghosh A, Pareek A, Sopory SK, Singla-Pareek SL. A glutathione responsive rice glyoxalase II, OsGLYII-2, functions in salinity adaptation by maintaining better photosynthesis efficiency and anti-oxidant pool. Plant J. 2014;80(1): 93-105.

10. Singla-Pareek S, Yadav S, Pareek A, Reddy M, Sopory S. Enhancing salt tolerance in a crop plant by overexpression of glyoxalase II. Transgenic Res. 2008;17(2):171-80
11. Kaur C, Mustafiz A, Sarkar AK, Ariyadasa TU, Singla-Pareek SL, Sopory SK. Expression of abiotic stress inducible ETHE1-like protein from rice is higher in roots and is regulated by calcium. Physiol Plant. 2014;152(1):1-16.

12. Mustafiz A, Ghosh A, Tripathi AK, Kaur C, Ganguly AK, Bhavesh NS, Tripathi JK, Pareek A, Sopory SK, Singla-Pareek SL. A unique Ni -dependent and methylglyoxal-inducible rice glyoxalase I possesses a single active site and functions in abiotic stress response. Plant J. 2014;78:951-63.

13. Mainali HR, Chapman P, Dhaubhadel S. Genome-wide analysis of Cyclophilin gene family in soybean (Glycine max). BMC Plant Biol. 2014;14:282.

14. Schmutz J, Cannon SB, Schlueter J, Ma J, Mitros T, Nelson W, Hyten DL, Song Q, Thelen JJ, Cheng J, et al. Genome sequence of the palaeopolyploid soybean. Nature. 2010;463(7278):178-83.

15. Manavalan LP, Guttikonda SK, Tran LS, Nguyen HT. Physiological and molecular approaches to improve drought resistance in soybean. Plant Cell Physiol. 2009;50(7):1260-76.

16. Singleton PW, Bohlool BB. Effect of salinity on nodule formation by soybean. Plant Physiol. 1984;74(1):72-6.

17. Chen X, Chen Z, Zhao H, Zhao Y, Cheng B, Xiang Y. Genome-wide analysis of soybean HD-Zip gene family and expression profiling under salinity and drought treatments. PLoS One. 2014;9(2), e87156.

18. Du H, Yang SS, Liang Z, Feng BR, Liu L, Huang YB, Tang YX. Genome-wide analysis of the MYB transcription factor superfamily in soybean. BMC Plant Biol. 2012:12:106.

19. Fan CM, Wang X, Wang YW, Hu RB, Zhang XM, Chen JX, Fu YF. Genomewide expression analysis of soybean MADS genes showing potential function in the seed development. PLoS One. 2013:8(4), e62288.

20. Xu H, Li Y, Yan Y, Wang K, Gao Y, Hu Y. Genome-scale identification of soybean BURP domain-containing genes and their expression under stress treatments. BMC Plant Biol. 2010;10:197

21. Yin $G, X u H$, Xiao S, Qin Y, Li Y, Yan Y, Hu Y. The large soybean (Glycine max) WRKY TF family expanded by segmental duplication events and subsequent divergent selection among subgroups. BMC Plant Biol. 2013:13:148.

22. Zhang G, Chen M, Chen X, Xu Z, Guan S, Li LC, Li A, Guo J, Mao L, Ma Y. Phylogeny, gene structures, and expression patterns of the ERF gene family in soybean (Glycine max L.). J Exp Bot. 2008;59(15):4095-107.

23. Yu CS, Chen YC, Lu CH, Hwang JK. Prediction of protein subcellular localization. Proteins. 2006;64(3):643-51.

24. Horton P, Park KJ, Obayashi T, Fujita N, Harada H, Adams-Collier CJ, Nakai K. WoLF PSORT: protein localization predictor. Nucleic Acids Res. 2007;35(Web Server issue):W585-7.

25. Emanuelsson $\mathrm{O}$, Nielsen $\mathrm{H}$, von Heijne G. ChloroP, a neural network-based method for predicting chloroplast transit peptides and their cleavage sites. Protein Sci. 1999:8(5):978-84

26. Lee $\mathrm{TH}$, Tang $\mathrm{H}$, Wang $\mathrm{X}$, Paterson AH. PGDD: a database of gene and genome duplication in plants. Nucleic Acids Res. 2013:41(Database issue):D1152-8.

27. Li WH, Gojobori T, Nei M. Pseudogenes as a paradigm of neutral evolution. Nature. 1981:292(5820):237-9.

28. Juretic N, Hoen DR, Huynh ML, Harrison PM, Bureau TE. The evolutionary fate of MULE-mediated duplications of host gene fragments in rice. Genome Res. 2005;15(9):1292-7.

29. Lynch M, Conery JS. The evolutionary fate and consequences of duplicate genes. Science. 2000;290(5494):1151-5.

30. Marasinghe GP, Sander IM, Bennett B, Periyannan G, Yang KW, Makaroff CA, Crowder MW. Structural studies on a mitochondrial glyoxalase II. J Biol Chem. 2005:280(49):40668-75.

31. Pesole G, Mignone F, Gissi C, Grillo G, Licciulli F, Liuni S. Structural and functional features of eukaryotic mRNA untranslated regions. Gene. 2001; 276(1-2):73-81.

32. Carvalho AB, Clark AG. Intron size and natural selection. Nature. 1999; 401(6751):344.

33. Fedorov A, Merican AF, Gilbert W. Large-scale comparison of intron positions among animal, plant, and fungal genes. Proc Natl Acad Sci U S A. 2002;99(25):16128-33.

34. Frickel EM, Jemth $P$, Widersten M, Mannervik B. Yeast glyoxalase I is a monomeric enzyme with two active sites. J Biol Chem. 2001;276(3):1845-9.

35. Deponte M, Sturm N, Mittler S, Harner M, Mack H, Becker K. Allosteric coupling of two different functional active sites in monomeric Plasmodium falciparum glyoxalase I. J Biol Chem. 2007:282(39):28419-30.

36. He MM, Clugston SL, Honek JF, Matthews BW. Determination of the structure of Escherichia coli glyoxalase I suggests a structural basis for differential metal activation. Biochemistry. 2000;39(30):8719-27. 
37. Cameron AD, Olin B, Ridderstrom M, Mannervik B, Jones TA. Crystal structure of human glyoxalase I-evidence for gene duplication and 3D domain swapping. EMBO J. 1997;16(12):3386-95.

38. Aronsson AC, Marmstal E, Mannervik B. Glyoxalase I, a zinc metalloenzyme of mammals and yeast. Biochem Biophys Res Commun. 1978;81(4):1235-40.

39. Ridderstrom M, Mannervik B. Optimized heterologous expression of the human zinc enzyme glyoxalase I. Biochem J. 1996;314(Pt 2):463-7.

40. Saint-Jean AP, Phillips KR, Creighton DJ, Stone MJ. Active monomeric and dimeric forms of Pseudomonas putida glyoxalase I: evidence for 3D domain swapping. Biochemistry. 1998;37(29):10345-53.

41. Ridderstrom M, Cameron AD, Jones TA, Mannervik B. Involvement of an active-site Zn2+ ligand in the catalytic mechanism of human glyoxalase I. J Biol Chem. 1998;273(34):21623-8.

42. Campos-Bermudez VA, Leite NR, Krog R, Costa-Filho AJ, Soncini FC, Oliva G, Vila AJ. Biochemical and structural characterization of Salmonella typhimurium glyoxalase II: new insights into metal ion selectivity. Biochemistry. 2007:46(39):11069-79.

43. Turra GL, Agostini RB, Fauguel CM, Presello DA, Andreo CS, Gonzalez JM, Campos-Bermudez VA. Structure of the novel monomeric glyoxalase I from Zea mays. Acta Crystallogr D Biol Crystallogr. 2015;71(Pt 10):2009-20.

44. Lescot M, Dehais P, Thijs G, Marchal K, Moreau Y, Van de Peer Y, Rouze P, Rombauts $\mathrm{S}$. PlantCARE, a database of plant cis-acting regulatory elements and a portal to tools for in silico analysis of promoter sequences. Nucleic Acids Res. 2002;30(1):325-7.

45. Chen W, Provart NJ, Glazebrook J, Katagiri F, Chang HS, Eulgem T, Mauch F, Luan S, Zou G, Whitham SA, et al. Expression profile matrix of Arabidopsis transcription factor genes suggests their putative functions in response to environmental stresses. Plant Cell. 2002;14(3):559-74.

46. Yamaguchi-Shinozaki K, Shinozaki K. Organization of cis-acting regulatory elements in osmotic- and cold-stress-responsive promoters. Trends Plant Sci. 2005;10(2):88-94.

47. Gu Z, Steinmetz LM, Gu X, Scharfe C, Davis RW, Li WH. Role of duplicate genes in genetic robustness against null mutations. Nature. 2003;421(6918):63-6.

48. Gu X. Functional divergence in protein (family) sequence evolution. Genetica. 2003;118(2-3):133-41.

49. Pakhomova S, Rife CL, Armstrong RN, Newcomer ME. Structure of fosfomycin resistance protein FosA from transposon Tn2921. Protein Sci. 2004;13(5):1260-5.

50. Skipsey M, Andrews CJ, Townson JK, Jepson I, Edwards R. Cloning and characterization of glyoxalase I from soybean. Arch Biochem Biophys. 2000; 374(2):261-8.

51. Suttisansanee U, Lau K, Lagishetty S, Rao KN, Swaminathan S, Sauder JM, Burley SK, Honek JF. Structural variation in bacterial glyoxalase I enzymes: investigation of the metalloenzyme glyoxalase I from Clostridium acetobutylicum. J Biol Chem. 2011;286(44):38367-74.

52. Limphong P, Nimako G, Thomas PW, Fast W, Makaroff CA, Crowder MW. Arabidopsis thaliana mitochondrial glyoxalase 2-1 exhibits beta-lactamase activity. Biochemistry. 2009;48(36):8491-3.

53. Grant D, Nelson RT, Cannon SB, Shoemaker RC. SoyBase, the USDA-ARS soybean genetics and genomics database. Nucleic Acids Res. 2010; 38(Database issue):D843-6.

54. Larkin MA, Blackshields G, Brown NP, Chenna R, McGettigan PA, McWilliam H, Valentin F, Wallace IM, Wilm A, Lopez R et al. Clustal W and Clustal X version 2. 0. Bioinformatics. 2007;23(21):2947-8.

55. Tamura K, Peterson D, Peterson N, Stecher G, Nei M, Kumar S. MEGA5: molecular evolutionary genetics analysis using maximum likelihood, evolutionary distance, and maximum parsimony methods. Mol Biol Evol. 2011;28(10):2731-9.

56. Suyama M, Torrents D, Bork P. PAL2NAL: robust conversion of protein sequence alignments into the corresponding codon alignments. Nucleic Acids Res. 2006;34(Web Server issue):W609-12

57. Biasini M, Bienert S, Waterhouse A, Arnold K, Studer G, Schmidt T, Kiefer F, Cassarino TG, Bertoni M, Bordoli L et al. SWISS-MODEL: modelling protein tertiary and quaternary structure using evolutionary information. Nucleic Acids Res. 2014;42(Web Server issue):W252-8.

58. Pettersen EF, Goddard TD, Huang CC, Couch GS, Greenblatt DM, Meng EC, Ferrin TE. UCSF Chimera-a visualization system for exploratory research and analysis. J Comput Chem. 2004;25(13):1605-12.

59. Eisen MB, Spellman PT, Brown PO, Botstein D. Cluster analysis and display of genome-wide expression patterns. Proc Natl Acad Sci U S A. 1998;95(25):14863-8.

60. Barrett T, Edgar R. Gene expression omnibus: microarray data storage, submission, retrieval, and analysis. Methods Enzymol. 2006;411:352-69.

\section{Submit your next manuscript to BioMed Central and we will help you at every step:}

- We accept pre-submission inquiries

- Our selector tool helps you to find the most relevant journal

- We provide round the clock customer support

- Convenient online submission

- Thorough peer review

- Inclusion in PubMed and all major indexing services

- Maximum visibility for your research

Submit your manuscript at www.biomedcentral.com/submit
C Biomed Central 